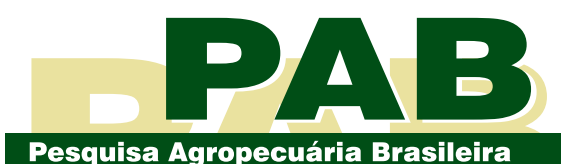

ISSN 1678-3921

Journal homepage: www.embrapa.br/pab

For manuscript submission and journal contents, access: www.scielo.br/pab

\title{
Physiological and productive traits of soybean with metsulfuron-methyl application at early desiccation
}

\begin{abstract}
The objective of this work was to evaluate the effects of metsulfuron-methyl rates, applied at different times, on the physiological and productive traits of soybean (Glycine max) in two crop years, in field conditions, using cucumber (Cucumis sativus) as a bioindicator plant, in greenhouse conditions. The experiments were conducted in a randomized complete block, in a $4 \times 5$ factorial arrangement, with four replicates. Factor A was the herbicide application time $(45,30,15$, and 0 days before soybean sowing, DBS), and factor B was the metsulfuron-methyl rate (0, 3.6, 5.4, 7.2, and $9.0 \mathrm{~g} \mathrm{ha}^{-1}$ a.i.). The following variables were evaluated: phytotoxicity, gas exchange, chlorophyll $a$ fluorescence, and yield components. The highest phytotoxicity in soybean plants is observed after the use of the highest rate of metsulfuron-methyl and with sowing at 0 DBS. The electron transport rate drastically reduces with an increasing metsulfuron-methyl rate and sowing proximity, whereas non-photochemical quenching, acting as a defense mechanism, increases in soybean plants exposed to a herbicide carryover of up to $5.4 \mathrm{~g} \mathrm{ha}^{-1}$. Metsulfuron-methyl carryover reduces soybean 1,000-grain weight and productivity as a function of application rate and sowing period. For the cucumber plants, negative effects on physiological traits were also verified. An interval of more than 45 days is recommended for sowing soybean after metsulfuron-methyl application for desiccation.
\end{abstract}

Universidade Federal da Fronteira Sul,
Laboratório de Manejo Sustentável dos Sistemas Agrícolas, ERS-135, Km 72, no 200, CEP 99700-970 Erechim, RS, Brazil. E-mail: jessicadiasgomes@hotmail.com, leandro.galone@gmail.com, renanpawelkiewicz17@gmail.com, milena.barretta@hotmail.com, julianec.portes@outlook.com, emanuelrossetto2011@gmail.com, gismaelperin@gmail.com, carolinemulleram@gmail.com

$\bowtie$ Corresponding author

Received

March 23, 2021

Accepted

July 05, 2021

How to cite

SILVA, J.D.G. da; GALON, L.; PAWELKIEWICZ, R.; FRANCESCHETTI, M.B.; PORTES, J.C.; ROSSETTO, E.R. de O.; PERIN, G.F.; MÜLLER, C. Physiological and productive traits of soybean with metsulfuron-methyl application at early desiccation. Pesquisa Agropecuária Brasileira, v.56, e02496, 2021. DOI: https://doi. org/10.1590/S1678-3921.pab2021.v56.02496.

Index terms: Cucumis sativus, Glycine max, herbicide, phytotoxicity.

\section{Características fisiológicas e produtivas de soja com aplicação de metsulfurom- metílico na dessecação precoce}

Resumo - O objetivo deste trabalho foi avaliar os efeitos de doses de metsulfurom-metílico, aplicadas em diferentes épocas, sobre as características fisiológicas e produtivas da soja (Glycine max), em dois anos agrícolas, em condições de campo, tendo-se utilizado o pepino (Cucumis sativus) como planta bioindicadora, em casa de vegetação. Os experimentos foram conduzidos em blocos ao acaso, em arranjo fatorial 4x5, com quatro repetições. $\mathrm{O}$ fator $\mathrm{A}$ foi o tempo de aplicação do herbicida $(45,30,15$ e 0 dias antes da semeadura da soja, DAS), e o fator B foi a dose de metsulfurommetílico ( $\left(0,3,6,5,4,7,2\right.$ e 9,0 $\mathrm{g} \mathrm{ha}^{-1}$ i.a.). Foram avaliadas as seguintes variáveis: fitotoxicidade, trocas gasosas, fluorescência da clorofila $a$ e componentes de rendimento. A maior fitotoxicidade em plantas de soja é observada após o uso da maior dose de metsulfurom-metílico e com semeadura em 0 DAS. A taxa de transporte de elétrons reduz drasticamente com o aumento da dose de metsulfurom-metílico e da proximidade da semeadura, enquanto a extinção 
não fotoquímica, como mecanismo de defesa, aumenta em plantas de soja expostas ao transporte de herbicida de até 5,4 $\mathrm{g} \mathrm{ha}^{-1}$. Os resíduos de metsulfurom-metílico reduzem o peso de mil grãos e a produtividade de soja em função da dose de aplicação e do período de semeadura. Para as plantas de pepino, também foram verificados efeitos negativos nas características fisiológicas. Um intervalo de mais de 45 dias é recomendado para a semeadura da soja após a aplicação de metsulfurom-metílico para dessecação.

Termos para indexação: Cucumis sativus, Glycine max, herbicida, fitotoxicidade.

\section{Introduction}

The no-tillage system has been widely used in recent years due to advantages over the conventional system, such as reduced erosion, better structured soil, increased water retention capacity, greater accumulation of organic matter, carbon sequestration in the soil, and greater biodiversity (Perotti et al., 2020). In addition, the viability of this system is causally related to the use of herbicides for weed management and crop desiccation, with glyphosate being the main product used (Benbrook, 2016).

The effectiveness in weed control and widespread use of glyphosate has allowed it to replace other herbicides in agricultural systems, with the possibility of a reduced number of applications, which has favored crop conservation and decreased the demand for alternative herbicides with a greater toxicity (Gilbert, 2013).

However, the dependence on glyphosate as the main method for weed management has caused resistance problems, resulting from inadequate management practices and an increase in the number of applications (Duke, 2012). This is interesting considering that one of the main challenges in chemical weed management has been the evolution of resistant weeds. Weed resistance is responsible for productivity losses, reduced commodity prices, lower land value, and increased management costs (Norsworthy et al., 2012). Furthermore, the use of transgenic crops resistant to herbicides has expanded, while the development of new herbicide molecules, particularly with new mechanisms of action, has decreased in the agrochemical industry (Duke, 2012).

In the no-tillage system, the most suitable method for controlling weeds is management during the offseason and pre-sowing period (Placido et al., 2015).
Early desiccation results in a greater yield in the sowing operation, a lower infestation of crops established in succession, and a higher efficiency of the herbicides applied after emergence (Lamego et al., 2013; Matos et al., 2016).

In this context, the adoption of practices for weed control allows slowing down the evolution of resistance to herbicides. Among the proposed alternatives, stand out herbicide rotation with different mechanisms of action and a residual effect (Santos et al., 2016), as well as soil cover, crop rotation, and the use of quality seed, among others. Herbicides with a longer activity period in the soil provide weed control for a longer time, suppressing new flows and reducing the number of necessary applications (Oliveira et al., 2018). However, herbicide residues in the soil can cause phytotoxicity and affect the growth and development of a crop grown in succession, a process known as carryover (Melo et al., 2016). This can occur with herbicides used for weed control in winter crops and in the management of desiccation, such as metsulfuron-methyl, which can cause damage to subsequent crops if the interval between their application and sowing is not respected, mainly for sensitive species (Rodrigues \& Almeida, 2018).

The herbicide metsulfuron-methyl is one of the most used from the group of sulfonylureas, due to its low toxicity to mammals, good selectivity for cultures, and recommendation for application at low rates (Yang et al., 2018). Like other sulfonylurea herbicides, metsulfuron-methyl acts by inhibiting the enzyme acetolactate synthase (ALS), which is responsible for catalyzing two parallel reactions in the biosynthetic pathway of the branched-chain amino acids valine, leucine, and isoleucine that are essential for the growth of plants (Ntoanidou et al., 2016). Producers have used this herbicide for early desiccation to reduce the density of species that are difficult to control, such as grove (Conyza spp.), and most of them sow soybean [Glycine max (L.) Merr.] after the use of metsulfuronmethyl. Although the interval recommended between the application of metsulfuron-methyl and sowing of soybean is of at least 60 days (Rodrigues \& Almeida, 2018), it may differ according to soil characteristics, climate, management, and tolerance of the soybean cultivars. It should be noted that the possibility of using the herbicide in a period closer to soybean sowing would be important to reduce the incidence

Pesq. agropec. bras., Brasília, v.56, e02496, 2021

DOI: 10.1590/S1678-3921.pab2021.v56.02496 
of weeds that develop in the off-season and infest the subsequent crop during its emergence.

The objective of this work was to evaluate the effects of metsulfuron-methyl rates, applied at different times, on the physiological and productive traits of soybean in two crop years, in field conditions, using cucumber as a bioindicator plant, in greenhouse conditions.

\section{Materials and Methods}

Two experiments were carried out in the experimental area of Universidade Federal da Fronteira Sul, located in the municipality of Erechim, in the state of Rio Grande do Sul, Brazil (27\%43'47"S, $52^{\circ} 17^{\prime} 37^{\prime \prime W}$, at $670 \mathrm{~m}$ altitude), in the 2017/2018 and $2018 / 2019$ crop years. The climate of the region is of the Cfa type, humid subtropical, according to the Köppen-Geiger classification (Peel et al., 2007), with well-distributed rains throughout the year and hot summers and cold winters. The soil was classified as a Latossolo Vermelho húmico aluminoférrico (Santos et al., 2018b), i.e., a Humic Aluminoferric Ferralsol, with the following physicochemical characteristics: $\mathrm{pH} 4.7$ in water, $3.04 \%$ organic matter, $8.5 \mathrm{mg} \mathrm{dm}^{-3} \mathrm{P}, 106$ $\mathrm{mg} \mathrm{dm}{ }^{-3} \mathrm{~K}, 1.0 \mathrm{cmol}_{\mathrm{c}} \mathrm{dm}^{-3} \mathrm{Al}^{3+}, 5.1 \mathrm{cmol}_{\mathrm{c}} \mathrm{dm}^{-3} \mathrm{Ca}^{2+}, 3.4$ $\mathrm{cmol}_{\mathrm{c}} \mathrm{dm}^{-3} \mathrm{Mg}^{2+}$, effective cation exchange capacity (CEC) of $9.9 \mathrm{cmol}_{\mathrm{c}} \mathrm{dm}^{-3}, \mathrm{CEC}_{\mathrm{pH} 7}$ of $18.6 \mathrm{cmol}_{\mathrm{c}} \mathrm{dm}^{-3}$,
$9.7 \mathrm{cmol}_{\mathrm{c}} \mathrm{dm}^{-3} \mathrm{H}+\mathrm{Al}$, base saturation of $48 \%$, and 640 $\mathrm{g} \mathrm{kg}^{-1}$ clay. The environmental conditions observed during the experimental period in the two crop years are shown in Figure 1.

The used experimental design was randomized complete blocks, arranged in a $4 \times 5$ factorial arrangement, with four replicates. In factor $\mathrm{A}$, the application time of metsulfuron-methyl was tested at $45,30,15$, and 0 days before sowing of soybean (DBS), and, in factor $\mathrm{B}$, the following herbicide rates were tested: $0,3.6,5.4,7.2$, and $9.0 \mathrm{~g} \mathrm{ha}^{-1}$ a.i. Each experimental unit occupied an area of $15 \mathrm{~m}^{2}(5.0 \times 3.0$ $\mathrm{m})$. In the winter period, the area was cultivated with black oat (Avena strigosa Schreb.) as a cover crop, in order to simulate the formation of straw in the no-tillage system. Prior to the installation of the experiments, the area was desiccated, providing 7,720.35, 5,431.5, $4,417.75$, and $3,483.7 \mathrm{~kg} \mathrm{ha}^{-1}$ dry matter in $2017 / 2018$ and 7,226.0, 5,154.8, 4,273.2, and 4,221.2 $\mathrm{kg} \mathrm{ha}^{-1}$ in 2018/2019 for herbicide applications at 45, 30, 15, and 0 DBS, respectively.

The metsulfuron-methyl herbicide (FMC Química do Brasil Ltda., Campinas, SP, Brazil) was applied using a backpack sprayer pressurized with $\mathrm{CO}_{2}$, equipped with the DG 110.02 four-spray boom (Névoa Comercial, Campinas, SP, Brazil), spaced at $50 \mathrm{~cm}$, and calibrated to provide a spray volume of $150 \mathrm{~L} \mathrm{ha}^{-1}$.

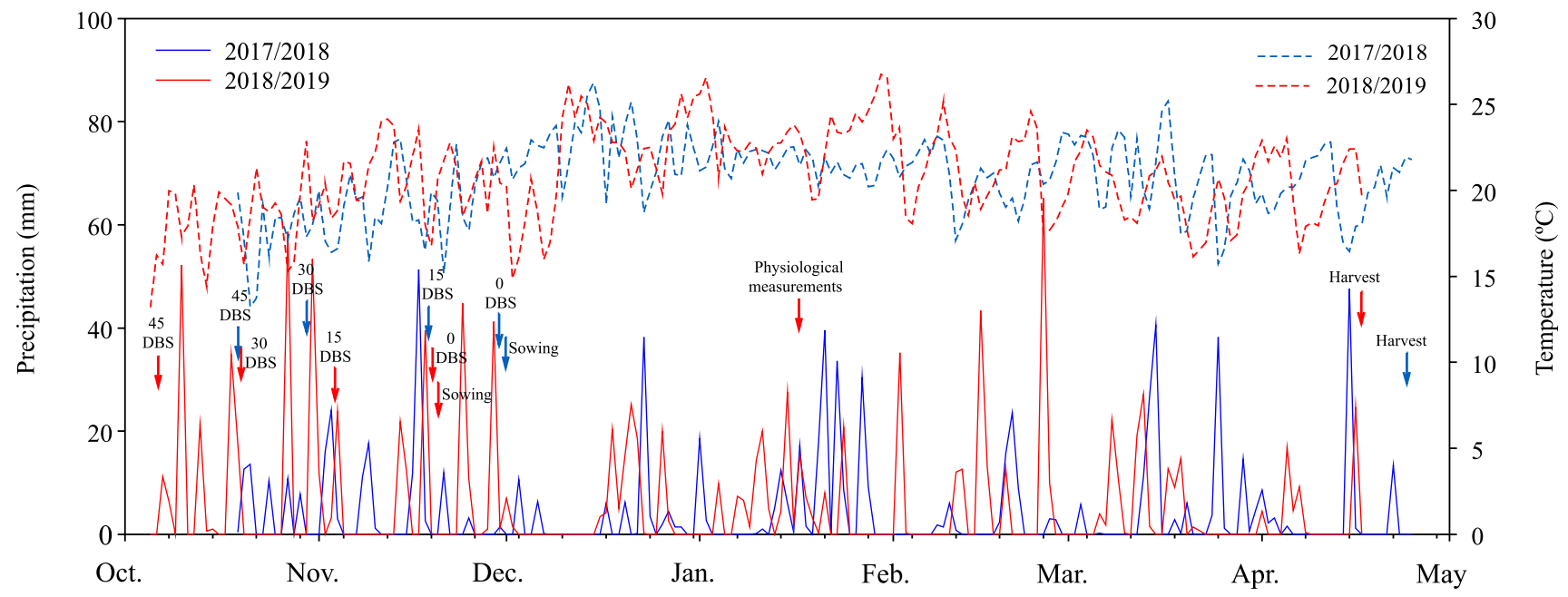

Figure 1. Average temperature and precipitation during the experimental periods, in two consecutive soybean (Glycine max) crop years (2017/2018 and 2018/2019), in the municipality of Erechim, in the state of Rio Grande do Sul, Brazil. DBS, days before soybean sowing. 
The soybean cultivar used in the 2017/2018 and 2018/2019 crop years was NA 5909 RG, which was sown under a no-tillage system. For sowing, a seeder/ fertilizer machine with six lines and $50 \mathrm{~cm}$ spacing between lines was used, and a density of 280 thousand plants per hectare was adopted. Fertilization was carried out according to the soil analysis, following the technical recommendations for soybean cultivation (Manual..., 2016), using $350 \mathrm{~kg} \mathrm{ha}^{-1}$ of the $\mathrm{N}-\mathrm{P}_{2} \mathrm{O}_{5}-\mathrm{K}_{2} \mathrm{O}$ (05-20-20) fertilizer at sowing in both experiments. When necessary, the crop was managed according to these same recommendations.

In parallel, the effect of metsulfuron-methyl was evaluated on cucumber plants (Cucumis sativus L.) of the Pioneiro cultivar, under greenhouse conditions. The greenhouse had an automatic irrigation system, temperature of $25 \pm 5^{\circ} \mathrm{C}$, and relative humidity of $65 \pm 5 \%$, being covered with a $150 \mu \mathrm{m}$ transparent plastic. The plants were placed apart to avoid shading. The experimental design was randomized complete blocks, with four replicates. The soil used was obtained from the experimental field, showing the previously indicated characteristics. In factor A, the application time of metsulfuron-methyl (45, 30,15 , and 0 DBS) was tested, and, in factor B, the application rate $\left(0,3.6,5.4,7.2\right.$, and $9.0 \mathrm{~g} \mathrm{ha}^{-1}$ a.i.). Three cucumber seed were sown in a $0.5 \mathrm{dm}^{3}$ pot, constituting an experimental unit. The plants were grown for 21 days.

Phytotoxicity was assessed at 7, 14, 21, 28, and 35 days after emergence (DAE) for field experiments and at 7 and 14 DAE for the greenhouse experiment, using a percentage scale, where zero $(0 \%)$ represented no damage and one hundred (100\%) indicated all dead plants (Velini et al., 1995).

Gas exchange was determined using the LCA PRO infrared gas analyzer (Analytical Development Co. Ltd, Hoddesdon, UK), evaluated on the last completely expanded leaf at the vegetative development stage (V8). Photosynthetic rate $\left(\mu \mathrm{mol} \mathrm{m}^{-2} \mathrm{~s}^{-1}\right)$, stomatal conductance $\left(\mathrm{mol} \mathrm{m} \mathrm{m}^{-2} \mathrm{~s}^{-1}\right)$, transpiration $\left(\mathrm{mol} \mathrm{m} \mathrm{m}^{-2} \mathrm{~s}^{-1}\right)$, and the ratio between internal and external concentration of $\mathrm{CO}_{2}\left(\mathrm{C}_{\mathrm{i}} /\right.$

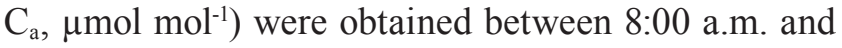
11:00 a.m. under environment photosynthetically active radiation $\left(\sim 1,000 \mu \mathrm{mol} \mathrm{m}{ }^{-2} \mathrm{~s}^{-1}\right), \mathrm{CO}_{2}$ concentration $\left(\mathrm{C}_{\mathrm{a}}\right.$, $\left.\sim 400 \mu \mathrm{mol} \mathrm{mol}{ }^{-1}\right)$, and temperature $\left(\sim 26.5^{\circ} \mathrm{C}\right)$.

Chlorophyll a fluorescence variables were determined using the OS5p portable modulated fluorometer (Opti-Sciences, Inc., Hudson, NH, USA). Leaves were initially adapted to the dark for $30 \mathrm{~min}$ to obtain initial fluorescence $\left(\mathrm{F}_{0}\right)$, maximum fluorescence $\left(\mathrm{F}_{\mathrm{m}}\right)$, and the potential quantum yield of photosystem II (PSII) $\left(\mathrm{F}_{\mathrm{v}} / \mathrm{F}_{\mathrm{m}}=\left(\mathrm{F}_{\mathrm{m}}-\mathrm{F}_{0}\right) / \mathrm{F}_{\mathrm{m}}\right)$. Leaves were exposed to illumination and a pulse of saturated actinic light to obtain: steady state fluorescence (F); light-adapted maximum fluorescence $\left(\mathrm{F}_{\mathrm{m}}\right.$ '); the effective quantum yield of PSII $\left(\mathrm{Y}_{\mathrm{II}}=\left(\mathrm{F}_{\mathrm{m}}{ }^{\prime}-\mathrm{F}\right) / \mathrm{F}_{\mathrm{m}}{ }^{\prime}\right)$; nonphotochemical quenching $\left(\mathrm{NPQ}=\left(\mathrm{F}_{\mathrm{m}}-\mathrm{F}_{\mathrm{m}}{ }^{\prime}\right) / \mathrm{F}_{\mathrm{m}}{ }^{\prime}\right)$; and the electron transport rate $\left(\mathrm{ETR}=\mathrm{Y}_{\mathrm{II}} \times \mathrm{PAR} \times 0.84 \times 0.5\right)$, where PAR was the flow of photon $\left(\mu \mathrm{mol} \mathrm{m} \mathrm{m}^{-2} \mathrm{~s}^{-1}\right)$ incidence on the leaves, 0.5 is the value corresponding to the fraction of excitation energy distributed to PSII, and 0.84 is the value corresponding to the fraction of incident light that is absorbed by leaves.

Plant material was collected from the greenhouse at 21 DAE. Cucumber shoots were collected, packed in paper bags, and dried in an oven with forced-air circulation at $65 \pm 5^{\circ} \mathrm{C}$ until the material reached a constant weight to obtain shoot dry matter.

The number of pods per plant and of grains per plant were evaluated in five plants collected randomly, in each experimental unit in the field. The trait 1,000-grain weight (g) was determined by extrapolation after counting samples of 100 grains for each replicate. Yield was evaluated from the grain harvest at $18 \%$ humidity in a useful area of 3.0 $\mathrm{m}^{2}$ for each experimental area. Grain moisture was corrected to $13 \%$, and yield data was extrapolated to $\mathrm{kg} \mathrm{ha}{ }^{-1}$.

The data obtained from the field experiments were subjected to the factorial analysis of variance by the F-test, at 5\% probability, and adjusted to response surfaces, where the herbicide rate was represented on the $\mathrm{X}$ axis, the soybean sowing interval after herbicide application was represented on the $\mathrm{Y}$ axis, and the analyzed variable was represented on the $\mathrm{Z}$ axis. For the greenhouse experiment, when significant differences were detected by the analysis of variance $(\mathrm{p} \leq 0.05)$, means were compared by Duncan's test, at $5 \%$ probability. All analyses were performed using the easyanova package of the $\mathrm{R}$, version 3.6.1, software (R Core Team, 2013), and graphs were plotted using the SigmaPlot, version 10.0, software (Systat Software Inc., San Jose, CA, USA). 


\section{Results and Discussion}

The occurrence of phytotoxicity was modeled according to the soybean sowing interval after the application of metsulfuron-methyl at different rates, and a response surface was evaluated over time in DAE, in the two crop years (Figure 2 and Table 1). Metsulfuron-methyl is active at very low concentrations and is characterized as slow acting, with a carryover potential that depends on the class and rate of the used herbicide, sensitivity of the crop in succession, soil characteristics, and environmental conditions before and after herbicide application (Ismail \& Azlizan, 2002; Santos et al., 2016; Gonçalves et al., 2018; Fraga et al., 2019).

A significant increase in phytotoxicity was observed throughout crop development, with more severe symptoms occurring at a shorter interval between herbicide application and soybean sowing. However, soybean plants in 2017/2018 (Figure 2 A, C, and E and Table 1) were more responsive to herbicide application than in 2018/2019, the following crop year (Figure 2 B, $\mathrm{D}$, and $\mathrm{F}$ and Table 1). Metsulfuron-methyl application at the highest rate caused a phytotoxicity of $40 \%$ at 7 DAE (Figure $2 \mathrm{~A}$ and Table 1), reaching $80 \%$ at 35 DAE (Figure 2 E and Table 1) in 2017/2018.

In both crop years, phytotoxicity increased more with the time than with the rate of application. Phytotoxicity was significantly reduced after 15 DBS, whose influence was greater than that of the herbicide rate itself. Leaf area was damaged by up to 83 and $77 \%$ in the 2017/2018 and 2018/2019 crop years, respectively, when metsulfuron-methyl was applied at 0 DBS (Table 1). Notably, when the proximity of sowing occurred together with a reduced presence of mulch, the highest rates of phytotoxicity caused by the herbicide were verified. However, this feature should be carefully evaluated. Although straw represents a barrier for the herbicide to reach the soil, it has been recommended to adopt herbicides with a greater solubility and a low vapor pressure and octanol-water partition coefficient (Matos et al., 2016), such as metsulfuron-methyl.

At $15 \mathrm{DBS}$, it was possible to observe a phytotoxicity of up to $30 \%$ in $2018 / 2019$, which gradually decreased with the increase in the interval between herbicide application and soybean sowing. In the same year, the lowest rate of the herbicide, together with the longest interval, resulted in 6.5\% leaf damage (Figure $2 \mathrm{~F}$ and Table 1). After the application of the herbicide at soybean pre-sowing, there was an accumulation of rain above 200 and $350 \mathrm{~mm}$ in 2017/2018 and 2018/2019, respectively (Figure 1), which may have contributed to the differences noted in the second crop year.

Conversely, the absence of precipitation during sowing and of good soil moisture favored herbicide uptake at 0 DBS, during the two crop years, which caused a high phytotoxicity and a high plant mortality. This result was further aggravated by a slight water deficit in the following two weeks after the emergence of the plants, which affected their metabolism, reduced their growth rate, and accentuated the damage caused to them during the second crop year.

Similarly, other authors have reported phytotoxicity due to the residual effects of ALS-inhibiting herbicides, both in corn (Zea mays L.), after 90 days of imazethapyr application (Santos et al., 2018a), and in soybean, after 445 days of imazapyr + imazapic application (Agostinetto et al., 2018). When studying the effects of the pre-emergent application of sulfentrazone on soybean, Aisenberg et al. (2016) found a phytotoxicity above $60 \%$ for the NA 5909 RG cultivar, which also affected its productivity. The authors emphasized that phytotoxicity is an important characteristic to be evaluated, even in selective cultures. In many cases, phytotoxicity (Figure 2 and Table 1) can affect yield components and, consequently, final crop yield, as was verified at the 0 DBS interval in both crop years.

Physiological data were modeled by response surfaces (Figure 3). Lower photosynthetic rates were observed at 0 and 45 DBS at all application rates (Figure $3 \mathrm{~A}$ and Table 2). Transpiration showed lower values in soybean plants grown when exposed to the highest rates of metsulfuron-methyl at 0 and 15 DBS (Figure $3 \mathrm{~B}$ and Table 2). In addition, the $\mathrm{C}_{\mathrm{i}} / \mathrm{C}_{\mathrm{a}}$ ratio was lower in soybean plants when the herbicide was applied at 15 and $30 \mathrm{DBS}$, regardless of the applied rate (Table 2). The lowest values of stomatal conductance were observed at the rate of $7.2 \mathrm{~g} \mathrm{ha}^{-1}$ at 0 DBS and at all rates at the other application intervals - from 15 to 45 DBS. Despite having no mode of action acting directly on photosystems, ALS-inhibiting herbicides have been described as having a secondary or indirect effect on plant physiological processes. A reduction in photosynthetic rates and in the quantum yield of PSII has been reported previously in different plants, such as wheat (Triticum aestivum L.) exposed to metsulfuronmethyl (Agostinetto et al., 2016) and sugarcane 

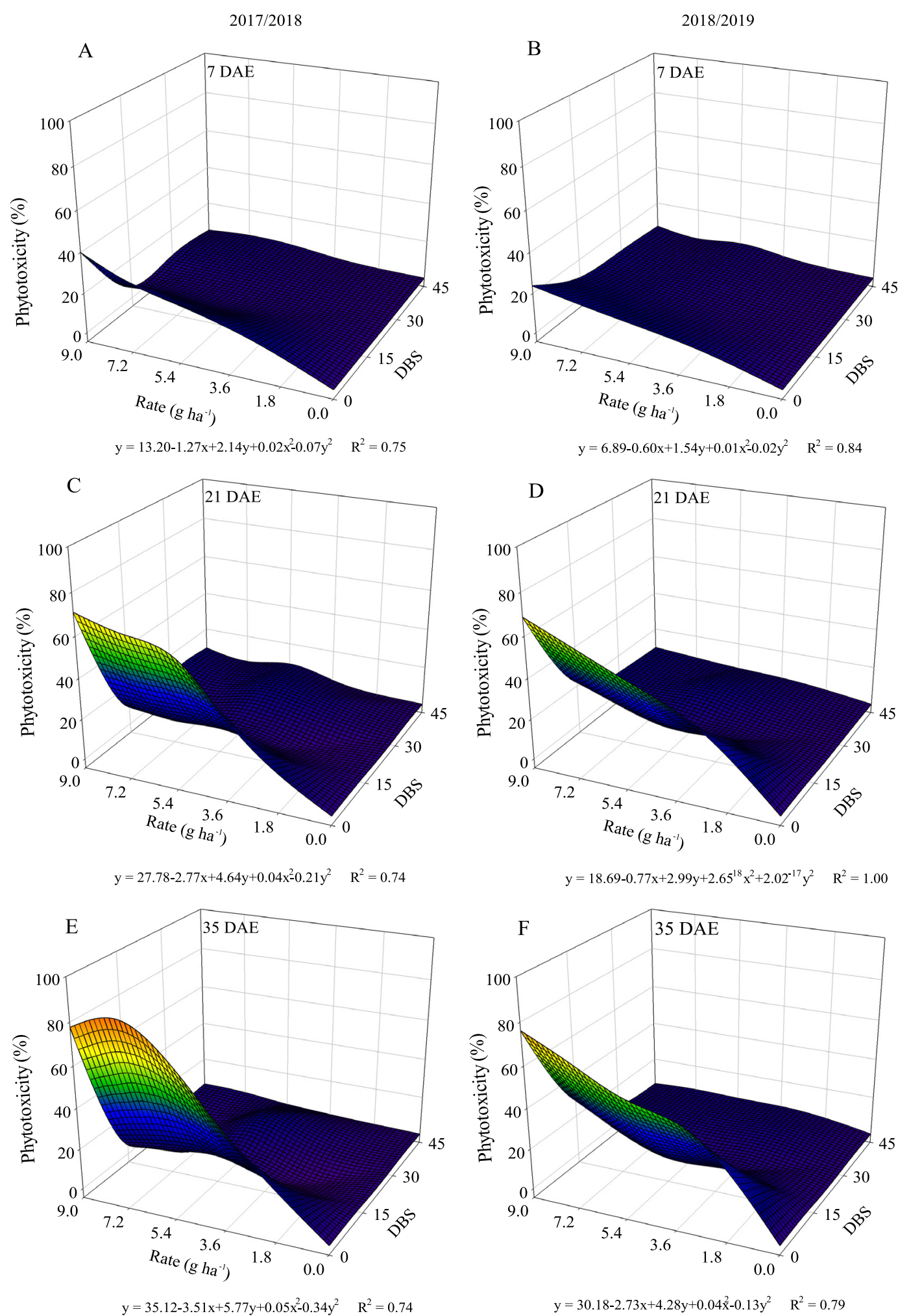

Figure 2. Phytotoxicity at 7 (A and B), 21 (C and D), and 35 (E and F) days after emergence (DAE) of the NA $5909 \mathrm{R}$ soybean (Glycine max) cultivar sown 45,30,15, and 0 days after the application of metsulfuron-methyl at different rates $(0$, 3.6, 5.4, 7.2, and $9.0 \mathrm{~g} \mathrm{ha}^{-1}$ ), in two consecutive crop years - 2017/2018 (A, C, and E) and 2018/2019 (B, D, and F) - in the municipality of Erechim, in the state of Rio Grande do Sul, Brazil. DBS, days before soybean sowing. 
(Saccharum spp.) exposed to trifloxysulfuron-sodium (Galon et al., 2010). The impairment of photosynthesis due to herbicide toxicity has been related to a lower $\mathrm{CO}_{2}$ fixation, plant development ( $\mathrm{Su}$ et al., 2018), and crop productivity (Gonçalves et al., 2018).

Associated with gas exchange traits, chlorophyll $a$ fluorescence is a quick and noninvasive tool for determining photosynthetic effects after herbicide exposure. The $F_{v} / F_{m}$ ratio had a slight reduction at 0 DBS at the highest rate, and photochemical quenching (qP) and the photochemical extinction coefficient (qL) were reduced by about $30 \%$ with the increase in metsulfuron-methyl rates, regardless of the application interval (Table 3). Changes in $\mathrm{q}_{\mathrm{P}}$ and $\mathrm{q}_{\mathrm{L}}$ indicate that

Table 1. Phytotoxicity at 7, 14, 21, 28, and 35 days after emergence (DAE) of the NA 5909 RG soybean (Glycine max) cultivar sown 45, 30, 15, and 0 days after the application of metsulfuron-methyl at different rates $(0,3.6,5.4,7.2$, and 9.0 $\mathrm{g} \mathrm{ha}^{-1}$ ), in two consecutive crop years (2017/2018 and 2018/2019), in the municipality of Erechim, in the state of Rio Grande do Sul, Brazil ${ }^{(1)}$.

\begin{tabular}{|c|c|c|c|c|c|c|c|c|c|c|c|}
\hline \multicolumn{2}{|l|}{ Factor } & \multicolumn{5}{|c|}{ Phytotoxicity (\%) - 2017/2018 crop year } & \multicolumn{5}{|c|}{ Phytotoxicity (\%) - 2018/2019 crop year } \\
\hline $\begin{array}{l}\text { Time } \\
(\mathrm{DBS})^{(2)}\end{array}$ & $\begin{array}{c}\text { Rate } \\
\left(\mathrm{g} \mathrm{ha}^{-1}\right. \\
\text { a.i. })\end{array}$ & $7 \mathrm{DAE}$ & $14 \mathrm{DAE}$ & $21 \mathrm{DAE}$ & $28 \mathrm{DAE}$ & $35 \mathrm{DAE}$ & $7 \mathrm{DAE}$ & $14 \mathrm{DAE}$ & $21 \mathrm{DAE}$ & $28 \mathrm{DAE}$ & $35 \mathrm{DAE}$ \\
\hline 45 & 0 & $0.0 \pm 0.0 \mathrm{aA}^{2}$ & $0.0 \pm 0.0 \mathrm{aA}$ & $0.0 \pm 0.0 \mathrm{aA}$ & $0.0 \pm 0.0 \mathrm{aA}$ & $0.0 \pm 0.0 \mathrm{aA}$ & $0.0 \pm 0.0 \mathrm{aD}$ & $0.0 \pm 0.0 \mathrm{aB}$ & $0.0 \pm 0.0 \mathrm{aB}$ & $0.0 \pm 0.0 \mathrm{aB}$ & $0.0 \pm 0.0 \mathrm{aA}$ \\
\hline 30 & 0 & $0.0 \pm 0.0 \mathrm{aA}$ & $0.0 \pm 0.0 \mathrm{aA}$ & $0.0 \pm 0.0 \mathrm{aA}$ & $0.0 \pm 0.0 \mathrm{aA}$ & $0.0 \pm 0.0 \mathrm{aA}$ & $0.0 \pm 0.0 \mathrm{aD}$ & $0.0 \pm 0.0 \mathrm{aC}$ & $0.0 \pm 0.0 \mathrm{aB}$ & $0.0 \pm 0.0 \mathrm{aC}$ & $0.0 \pm 0.0 \mathrm{aB}$ \\
\hline 15 & 0 & $0.0 \pm 0.0 \mathrm{aA}$ & $0.0 \pm 0.0 \mathrm{aA}$ & $0.0 \pm 0.0 \mathrm{aA}$ & $0.0 \pm 0.0 \mathrm{aA}$ & $0.0 \pm 0.0 \mathrm{aA}$ & $0.0 \pm 0.0 \mathrm{aE}$ & $0.0 \pm 0.0 \mathrm{aC}$ & $0.0 \pm 0.0 \mathrm{aC}$ & $0.0 \pm 0.0 \mathrm{aD}$ & $0.0 \pm 0.0 \mathrm{aD}$ \\
\hline 0 & 0 & $0.0 \pm 0.0 \mathrm{aD}$ & $0.0 \pm 0.0 \mathrm{aD}$ & $0.0 \pm 0.0 \mathrm{aC}$ & $0.0 \pm 0.0 \mathrm{aD}$ & $0.0 \pm 0.0 \mathrm{aC}$ & $0.0 \pm 0.0 \mathrm{aE}$ & $0.0 \pm 0.0 \mathrm{aD}$ & $0.0 \pm 0.0 \mathrm{aE}$ & $0.0 \pm 0.0 \mathrm{aE}$ & $0.0 \pm 0.0 \mathrm{aD}$ \\
\hline 45 & 3.6 & $2.0 \pm 1.2 \mathrm{bA}$ & $3.3 \pm 2.0 \mathrm{bA}$ & $4.0 \pm 2.3 \mathrm{bA}$ & $0.0 \pm 0.0 \mathrm{bA}$ & $1.5 \pm 0.9 \mathrm{bA}$ & $2.8 \pm 0.3 \mathrm{bC}$ & $6.5 \pm 0.9 \mathrm{bA}$ & $5.3 \pm 1.0 \mathrm{bAB}$ & $4.5 \pm 2.1 \mathrm{bAB}$ & $6.0 \pm 3.0 \mathrm{bA}$ \\
\hline 30 & 3.6 & $2.8 \pm 1.9 \mathrm{bA}$ & $1.7 \pm 1.2 \mathrm{bA}$ & $7.0 \pm 1.2 \mathrm{bA}$ & $5.8 \pm 2.2 \mathrm{bA}$ & $4.5 \pm 1.7 \mathrm{bA}$ & $3.0 \pm 0.0 \mathrm{bC}$ & $5.8 \pm 0.8 \mathrm{bB}$ & $5.8 \pm 0.8 \mathrm{bAB}$ & $4.0 \pm 0.6 \mathrm{bBC}$ & $5.8 \pm 0.8 \mathrm{bAB}$ \\
\hline 15 & 3.6 & $5.3 \pm 1.0 \mathrm{bA}$ & $5.0 \pm 0.0 \mathrm{bA}$ & $3.8 \pm 2.4 \mathrm{bA}$ & $6.5 \pm 2.2 \mathrm{bA}$ & $2.5 \pm 1.4 \mathrm{bA}$ & $3.8 \pm 0.5 b D$ & $7.8 \pm 1.0 \mathrm{bB}$ & $8.0 \pm 0.0 \mathrm{bB}$ & $7.0 \pm 1.2 \mathrm{bC}$ & $8.0 \pm 0.0 \mathrm{bC}$ \\
\hline 0 & 3.6 & $18.3 \pm 4.3 \mathrm{aC}$ & $18.8 \pm 5.2 \mathrm{aC}$ & $34.5 \pm 5.9 \mathrm{aB}$ & $42.5 \pm 7.8 \mathrm{aC}$ & $42.5 \pm 10.9 \mathrm{aB}$ & $12.0 \pm 0.0 \mathrm{aD}$ & $25.5 \pm 1.7 \mathrm{aC}$ & $38.8 \pm 3.8 \mathrm{aD}$ & $41.3 \pm 1.3 \mathrm{aD}$ & $47.5 \pm 1.4 \mathrm{aC}$ \\
\hline 45 & 5.4 & $4.8 \pm 1.2 \mathrm{bA}$ & $1.3 \pm 1.3 \mathrm{bA}$ & $8.8 \pm 1.3 \mathrm{bA}$ & $3.3 \pm 2.0 \mathrm{bA}$ & $2.8 \pm 1.0 \mathrm{bA}$ & $5.5 \pm 0.9 \mathrm{bAB}$ & $7.3 \pm 0.8 \mathrm{bA}$ & $6.3 \pm 1.3 \mathrm{bAB}$ & $6.0 \pm 1.2 \mathrm{bA}$ & $6.3 \pm 1.3 \mathrm{bA}$ \\
\hline 30 & 5.4 & $4.3 \pm 1.7 \mathrm{bA}$ & $3.0 \pm 3.0 \mathrm{bA}$ & $9.5 \pm 3.6 \mathrm{bA}$ & $3.8 \pm 2.4 \mathrm{bA}$ & $10.0 \pm 10.0 \mathrm{bA}$ & $5.3 \pm 0.9 \mathrm{bB}$ & $6.0 \pm 1.2 \mathrm{bB}$ & $9.5 \pm 1.0 \mathrm{bA}$ & $5.8 \pm 0.8 \mathrm{bBC}$ & $5.3 \pm 1.0 \mathrm{bAB}$ \\
\hline 15 & 5.4 & $5.3 \pm 2.3 \mathrm{bA}$ & $3.8 \pm 1.3 \mathrm{bA}$ & $7.5 \pm 3.2 \mathrm{bA}$ & $9.5 \pm 5.5 \mathrm{bA}$ & $8.8 \pm 5.9 \mathrm{bA}$ & $6.0 \pm 0.7 \mathrm{bC}$ & $9.5 \pm 0.5 \mathrm{bB}$ & $8.0 \pm 1.8 \mathrm{bB}$ & $10.0 \pm 0.8 \mathrm{bC}$ & $7.5 \pm 1.7 \mathrm{bC}$ \\
\hline 0 & 5.4 & $24.3 \pm 8.7 \mathrm{aBC}$ & $38.8 \pm 10.9 \mathrm{aB}$ & $58.8 \pm 12.1 \mathrm{aA}$ & $65.0 \pm 13.7 \mathrm{aB}$ & $68.5 \pm 15.0 \mathrm{aA}$ & $15.8 \pm 0.8 \mathrm{aC}$ & $28.8 \pm 3.1 \mathrm{aC}$ & $48.8 \pm 3.8 \mathrm{aC}$ & $50.0 \pm 4.6 \mathrm{aC}$ & $53.8 \pm 3.8 \mathrm{aC}$ \\
\hline 45 & 7.2 & $5.8 \pm 2.1 \mathrm{bA}$ & $7.5 \pm 3.2 \mathrm{bA}$ & $5.8 \pm 4.3 \mathrm{bA}$ & $10.7 \pm 5.2 \mathrm{bA}$ & $5.0 \pm 3.5 \mathrm{bA}$ & $3.8 \pm 0.5 \mathrm{cBC}$ & $10.0 \pm 0.8 \mathrm{bA}$ & $7.8 \pm 1.0 \mathrm{bA}$ & $7.3 \pm 1.5 \mathrm{cA}$ & $6.8 \pm 1.3 \mathrm{cA}$ \\
\hline 30 & 7.2 & $5.3 \pm 2.5 \mathrm{bA}$ & $3.8 \pm 2.4 \mathrm{bA}$ & $5.0 \pm 3.0 \mathrm{bA}$ & $5.8 \pm 2.2 \mathrm{bA}$ & $1.3 \pm 1.3 \mathrm{bA}$ & $5.5 \pm 0.9 \mathrm{cB}$ & $9.0 \pm 2.1 \mathrm{bAB}$ & $8.3 \pm 1.2 \mathrm{bA}$ & $7.8 \pm 1.0 \mathrm{cB}$ & $7.3 \pm 0.8 \mathrm{cA}$ \\
\hline 15 & 7.2 & $6.5 \pm 2.0 \mathrm{bA}$ & $7.5 \pm 3.2 \mathrm{bA}$ & $6.8 \pm 2.7 \mathrm{bA}$ & $10.0 \pm 5.0 \mathrm{bA}$ & $7.8 \pm 5.8 \mathrm{bA}$ & $8.5 \pm 0.5 \mathrm{bB}$ & $10.5 \pm 1.0 \mathrm{bAB}$ & $14.0 \pm 4.0 \mathrm{bB}$ & $17.8 \pm 3.1 \mathrm{bB}$ & $15.8 \pm 4.0 \mathrm{bB}$ \\
\hline 0 & 7.2 & $30.0 \pm 11.0 \mathrm{aAB}$ & $37.5 \pm 4.8 \mathrm{aB}$ & $65.0 \pm 7.9 \mathrm{aA}$ & $72.5 \pm 10.3 \mathrm{aAB}$ & $84.8 \pm 3.9 \mathrm{aA}$ & $19.5 \pm 0.5 \mathrm{aB}$ & $36.3 \pm 1.3 \mathrm{aB}$ & $58.8 \pm 2.4 \mathrm{aB}$ & $63.8 \pm 4.3 \mathrm{aB}$ & $65.0 \pm 4.1 \mathrm{aB}$ \\
\hline 45 & 9.0 & $4.5 \pm 0.5 \mathrm{bA}$ & $10.0 \pm 5.0 \mathrm{bA}$ & $9.3 \pm 1.5 \mathrm{bA}$ & $8.5 \pm 2.9 \mathrm{bA}$ & $5.8 \pm 3.3 \mathrm{bA}$ & $6.8 \pm 0.8 \mathrm{cA}$ & $7.8 \pm 1.0 \mathrm{cA}$ & $9.5 \pm 1.0 \mathrm{cA}$ & $7.5 \pm 1.4 \mathrm{dA}$ & $6.0 \pm 1.2 \mathrm{cA}$ \\
\hline 30 & 9.0 & $6.0 \pm 1.2 \mathrm{bA}$ & $2.0 \pm 2.0 \mathrm{bA}$ & $9.5 \pm 1.0 \mathrm{bA}$ & $4.3 \pm 2.8 \mathrm{bA}$ & $2.0 \pm 2.0 \mathrm{bA}$ & $8.5 \pm 0.5 \mathrm{cA}$ & $13.0 \pm 1.7 \mathrm{bA}$ & $11.0 \pm 0.6 \mathrm{cA}$ & $13.5 \pm 1.5 \mathrm{cB}$ & $8.8 \pm 1.3 \mathrm{cA}$ \\
\hline 15 & 9.0 & $5.8 \pm 2.1 \mathrm{bA}$ & $4.5 \pm 1.7 \mathrm{bA}$ & $8.8 \pm 1.5 \mathrm{bA}$ & $10.0 \pm 3.5 \mathrm{bA}$ & $4.5 \pm 2.1 \mathrm{bA}$ & $10.8 \pm 1.5 \mathrm{bA}$ & $14.3 \pm 1.4 \mathrm{bA}$ & $21.8 \pm 6.9 \mathrm{bA}$ & $30.0 \pm 3.5 \mathrm{bA}$ & $27.8 \pm 5.7 \mathrm{bA}$ \\
\hline 0 & 9.0 & $40.0 \pm 8.4 \mathrm{aA}$ & $51.3 \pm 12.1 \mathrm{aA}$ & $71.0 \pm 14.7 \mathrm{aA}$ & $83.0 \pm 7.7 \mathrm{aA}$ & $77.8 \pm 14.3 \mathrm{aA}$ & $23.8 \pm 1.3 \mathrm{aA}$ & $43.8 \pm 3.8 \mathrm{aA}$ & $68.8 \pm 1.3 \mathrm{aA}$ & $76.8 \pm 2.7 \mathrm{aA}$ & $76.3 \pm 4.7 \mathrm{aA}$ \\
\hline \multicolumn{2}{|l|}{45} & $3.4 \pm 0.7 b$ & $4.4 \pm 1.4 \mathrm{~b}$ & $5.6 \pm 1.2 b$ & $4.5 \pm 1.5 b$ & $3.0 \pm 1.0 \mathrm{~b}$ & $3.8 \pm 0.6 \mathrm{c}$ & $6.3 \pm 0.8 \mathrm{c}$ & $5.8 \pm 0.8 \mathrm{c}$ & $5.1 \pm 0.9 \mathrm{c}$ & $5.0 \pm 0.9 \mathrm{c}$ \\
\hline \multicolumn{2}{|c|}{30} & $3.7 \pm 0.8 b$ & $2.1 \pm 0.9 \mathrm{~b}$ & $6.2 \pm 1.2 \mathrm{~b}$ & $3.9 \pm 1.0 \mathrm{~b}$ & $3.6 \pm 2.0 \mathrm{~b}$ & $4.5 \pm 0.7 \mathrm{c}$ & $6.8 \pm 1.1 \mathrm{bc}$ & $6.9 \pm 0.9 \mathrm{c}$ & $6.2 \pm 1.1 \mathrm{c}$ & $5.4 \pm 0.8 \mathrm{c}$ \\
\hline \multicolumn{2}{|c|}{15} & $4.6 \pm 0.9 b$ & $4.2 \pm 0.9 \mathrm{~b}$ & $5.4 \pm 1.2 \mathrm{~b}$ & $7.2 \pm 1.8 \mathrm{~b}$ & $4.7 \pm 1.7 \mathrm{~b}$ & $5.8 \pm 0.9 \mathrm{~b}$ & $8.4 \pm 1.1 \mathrm{~b}$ & $10.4 \pm 2.2 \mathrm{~b}$ & $13.0 \pm 2.5 b$ & $11.8 \pm 2.5 b$ \\
\hline \multicolumn{2}{|l|}{0} & $22.5 \pm 4.3 \mathrm{a}$ & $29.3 \pm 5.2 \mathrm{a}$ & $45.9 \pm 7.1 \mathrm{a}$ & $52.6 \pm 7.7 \mathrm{a}$ & $54.7 \pm 8.2 \mathrm{a}$ & $14.2 \pm 1.9 \mathrm{a}$ & $26.9 \pm 3.5 \mathrm{a}$ & $43.0 \pm 5.5 \mathrm{a}$ & $46.4 \pm 6.1 \mathrm{a}$ & $48.5 \pm 6.2 \mathrm{a}$ \\
\hline \multicolumn{2}{|l|}{0} & $0.0 \pm 0.0 \mathrm{C}$ & $0.0 \pm 0.0 \mathrm{C}$ & $0.0 \pm 0.0 \mathrm{C}$ & $0.0 \pm 0.0 \mathrm{C}$ & $0.0 \pm 0.0 \mathrm{C}$ & $0.0 \pm 0.0 \mathrm{E}$ & $0.0 \pm 0.0 \mathrm{D}$ & $0.0 \pm 0.0 \mathrm{E}$ & $0.0 \pm 0.0 \mathrm{E}$ & $0.0 \pm 0.0 \mathrm{D}$ \\
\hline \multicolumn{2}{|c|}{3.6} & $7.1 \pm 2.0 \mathrm{~B}$ & $7.2 \pm 2.2 \mathrm{~B}$ & $12.3 \pm 3.7 \mathrm{~B}$ & $13.7 \pm 4.7 \mathrm{~B}$ & $12.8 \pm 5.1 \mathrm{~B}$ & $5.4 \pm 1.0 \mathrm{D}$ & $11.4 \pm 2.2 \mathrm{C}$ & $14.4 \pm 3.7 \mathrm{D}$ & $14.2 \pm 4.1 \mathrm{D}$ & $16.8 \pm 4.6 \mathrm{C}$ \\
\hline \multicolumn{2}{|c|}{5.4} & $9.6 \pm 3.0 \mathrm{AB}$ & $11.7 \pm 4.8 \mathrm{AB}$ & $21.1 \pm 6.3 \mathrm{~B}$ & $20.4 \pm 7.5 \mathrm{AB}$ & $22.5 \pm 8.1 \mathrm{~A}$ & $8.1 \pm 1.2 \mathrm{C}$ & $12.9 \pm 2.5 \mathrm{C}$ & $18.1 \pm 4.7 \mathrm{C}$ & $17.9 \pm 4.9 \mathrm{C}$ & $18.2 \pm 5.4 \mathrm{C}$ \\
\hline \multicolumn{2}{|c|}{7.2} & $11.9 \pm 3.8 \mathrm{AB}$ & $14.1 \pm 3.9 \mathrm{~A}$ & $20.6 \pm 7.0 \mathrm{~B}$ & $24.8 \pm 7.7 \mathrm{~A}$ & $24.7 \pm 9.2 \mathrm{~A}$ & $9.3 \pm 1.6 \mathrm{~B}$ & $16.4 \pm 3.0 \mathrm{~B}$ & $22.2 \pm 5.6 \mathrm{~B}$ & $24.1 \pm 6.1 \mathrm{~B}$ & $23.7 \pm 6.4 \mathrm{~B}$ \\
\hline \multicolumn{2}{|c|}{9.0} & $14.1 \pm 4.3 \mathrm{~A}$ & $16.9 \pm 6.0 \mathrm{~A}$ & $24.6 \pm 7.7 \mathrm{~A}$ & $26.4 \pm 8.7 \mathrm{~A}$ & $22.5 \pm 8.9 \mathrm{~A}$ & $12.4 \pm 1.8 \mathrm{~A}$ & $19.7 \pm 3.8 \mathrm{~A}$ & $27.8 \pm 6.4 \mathrm{~A}$ & $31.9 \pm 7.1 \mathrm{~A}$ & $29.7 \pm 7.5 \mathrm{~A}$ \\
\hline \multicolumn{12}{|c|}{ Variation source } \\
\hline \multicolumn{2}{|c|}{$\begin{array}{l}\text { Application } \\
\text { time }(\mathrm{T})\end{array}$} & $<0.001$ & $<0.001$ & $<0.001$ & $<0.001$ & $<0.001$ & $<0.001$ & $<0.001$ & $<0.001$ & $<0.001$ & $<0.001$ \\
\hline \multicolumn{2}{|l|}{ Rate (R) } & $<0.001$ & $<0.001$ & $<0.001$ & $<0.001$ & $<0.001$ & $<0.001$ & $<0.001$ & $<0.001$ & $<0.001$ & $<0.001$ \\
\hline \multicolumn{2}{|l|}{$\mathrm{T} \times \mathrm{R}$} & 0.0044 & $<0.001$ & $<0.001$ & $<0.001$ & $<0.001$ & $<0.001$ & $<0.001$ & $<0.001$ & $<0.001$ & $<0.001$ \\
\hline \multicolumn{2}{|l|}{ Block } & 0.03 & 0.0512 & 0.1043 & 0.0256 & 0.03 & 0.0863 & 0.2867 & 0.0782 & 0.0413 & 0.0226 \\
\hline \multicolumn{2}{|l|}{ CV (\%) } & 89.43 & 85.04 & 64.08 & 58.13 & 71.44 & 18.52 & 24.71 & 27.57 & 22.50 & 26.65 \\
\hline
\end{tabular}

${ }^{(1)}$ Means followed by equal letters, lowercase letters compare application times and uppercase letters compare the different rates applied, do not differ by Duncan's test, at $5 \%$ probability. ${ }^{(2)}$ Days before soybean sowing. $\mathrm{CV}$, coefficient of variation. 
a lower photochemical dissipation may be associated with a closed proportion of PSII reaction centers, that is, with an increase in the proportion of the reduced state of quinone $\mathrm{A}$ and a decrease in the excitation energy available for photochemical processes (Genty et al., 1989). In addition, the reaction centers are constituted by chlorophylls, which have already been reported as being reduced in soybean plants exposed to the carryover of imazapyr + imazapic, both ALSinhibiting herbicides (Fraga et al., 2019).

Even at 45 DBS, a reduction in ETR was observed at rates higher than $3.6 \mathrm{~g} \mathrm{ha}^{-1}$ (Figure $3 \mathrm{C}$ and Table 3 ). The impairment of PSII affects the ETR and the production of adenosine triphosphate and nicotinamide adenine dinucleotide phosphate necessary for Calvin cycle functioning and $\mathrm{CO}_{2}$ fixation (Timm et al., 2016). Fraga et al. (2019) also found an increase in lipid
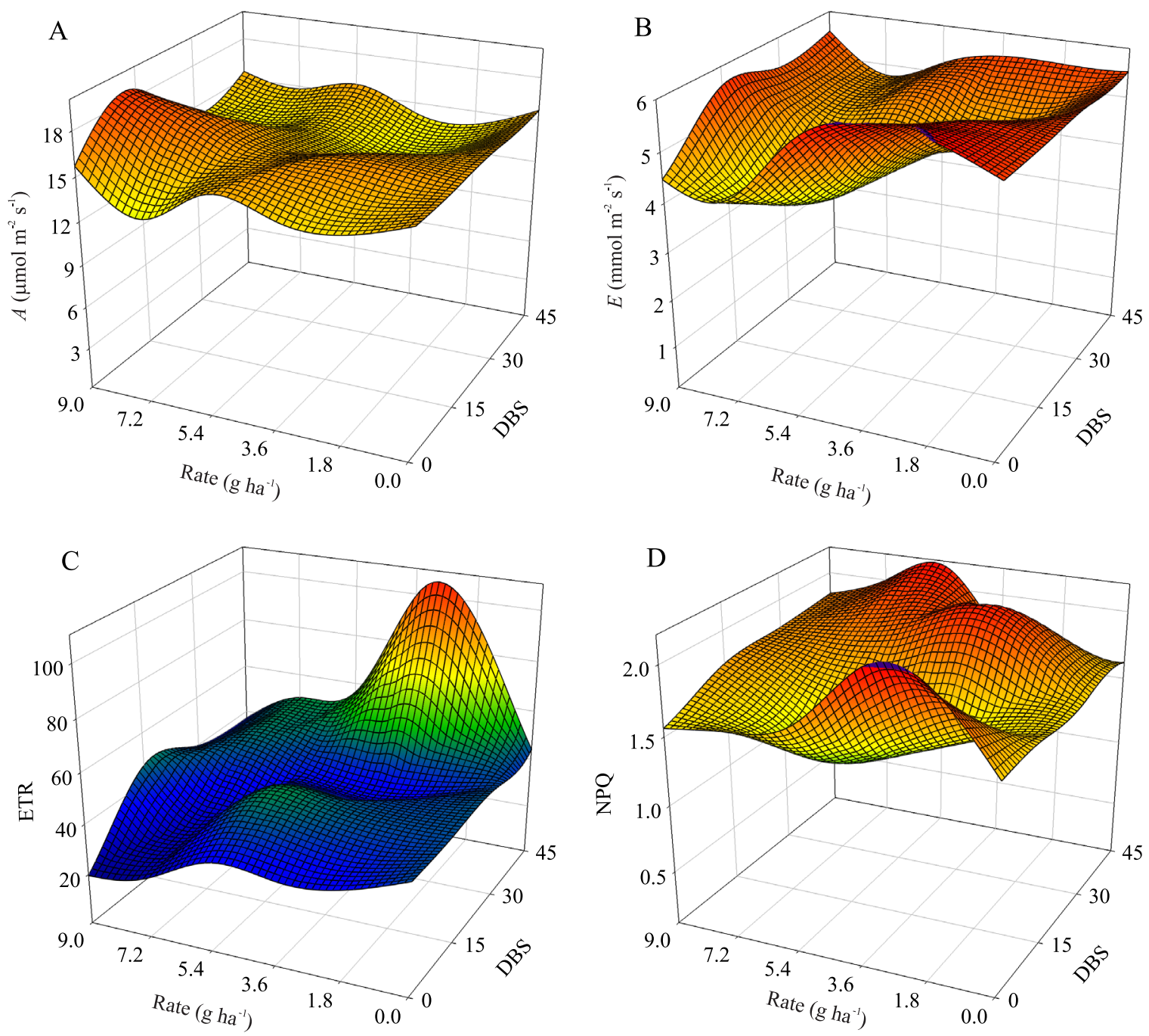

Figure 3. Photosynthetic rate $(A)(\mathrm{A})$, transpiration $(E)(\mathrm{B})$, electron transport rate (ETR) $(\mathrm{C})$, and nonphotochemical quenching (NPQ) (D) of the NA 5909 RG soybean (Glycine max) cultivar sown 45, 30, 15, and 0 days after the application of metsulfuron-methyl at different rates $\left(0,3.6,5.4,7.2\right.$, and $\left.9.0 \mathrm{~g} \mathrm{ha}^{-1}\right)$, in the 2018/2019 crop year, in the municipality of Erechim, in the state of Rio Grande do Sul, Brazil. DBS, days before soybean sowing. 
peroxidation in soybean leaves, which could cause oxidative stress and the degradation of the ribulose1,5-bisphosphate carboxylase/oxygenase (RuBisCO) enzyme, which fixes atmospheric carbon dioxide within photosynthesis (Das et al., 2016).

Nonphotochemical quenching was greater at 0 DBS than at the lowestapplication rates. Highvalues were also observed at 45 DBS, regardless of the applied herbicide rate (Figure $3 \mathrm{D}$ and Table 3). Nonphotochemical quenching acts as a stress suppression mechanism by the thermal dissipation of excess energy absorbed in PSII, through the xanthophyll cycle (Niyogi et al., 1997), aiming to avoid oxidative stress and reductions in photosynthetic rate, which is the primary electron sink, also reducing the potential quantum derived from photochemical reactions. However, the activation

Table 2. Photosynthetic rate $(A)$, stomatal conductance $\left(g_{\mathrm{S}}\right)$, transpiration $(E)$, and ratio between internal and external $\mathrm{CO}_{2}$ concentration $(\mathrm{Ci} / \mathrm{Ca})$ of the NA $5909 \mathrm{RG}$ soybean (Glycine max) cultivar sown 45, 30, 15, and 0 days after the application of metsulfuron-methyl at different rates $\left(0,3.6,5.4,7.2\right.$, and $\left.9.0 \mathrm{~g} \mathrm{ha}^{-1}\right)$, in the 2018/2019 crop year, in the municipality of Erechim, in the state of Rio Grande do Sul, Brazil ${ }^{(1)}$.

\begin{tabular}{|c|c|c|c|c|c|}
\hline \multicolumn{2}{|c|}{ Factor } & \multirow{2}{*}{$\begin{array}{c}A \\
\left(\mu \mathrm{mol} \mathrm{m}{ }^{-2} \mathrm{~s}^{-1}\right)\end{array}$} & \multirow{2}{*}{$\begin{array}{c}g_{S} \\
\left(\mathrm{~mol} \mathrm{~m}^{-2} \mathrm{~s}^{-1}\right)\end{array}$} & \multirow{2}{*}{$\begin{array}{c}E \\
\left(\mathrm{mmol} \mathrm{mol}^{-1}\right)\end{array}$} & \multirow{2}{*}{$\begin{array}{c}\mathrm{Ci} / \mathrm{Ca} \\
\left(\mu \mathrm{mol} \mathrm{mol}^{-1}\right)\end{array}$} \\
\hline $\begin{array}{l}\text { Application time } \\
\text { (DBS) }^{(2)}\end{array}$ & $\begin{array}{c}\text { Rate } \\
\left(\mathrm{g} \mathrm{ha}^{-1} \text { a.i. }\right)\end{array}$ & & & & \\
\hline 45 & 0 & $15.69 \pm 0.62 \mathrm{aA}^{2}$ & $1.37 \pm 0.14 \mathrm{aA}$ & $5.53 \pm 0.37 \mathrm{aA}$ & $0.827 \pm 0.022 \mathrm{aAB}$ \\
\hline 30 & 0 & $15.69 \pm 0.62 \mathrm{aA}$ & $1.37 \pm 0.14 \mathrm{aA}$ & $5.53 \pm 0.37 \mathrm{aA}$ & $0.827 \pm 0.022 \mathrm{aA}$ \\
\hline 15 & 0 & $15.69 \pm 0.62 \mathrm{aB}$ & $1.37 \pm 0.14 \mathrm{aA}$ & $5.53 \pm 0.37 \mathrm{aA}$ & $0.827 \pm 0.022 \mathrm{aA}$ \\
\hline 0 & 0 & $15.69 \pm 0.62 \mathrm{aA}$ & $1.37 \pm 0.14 \mathrm{aB}$ & $5.53 \pm 0.37 \mathrm{aA}$ & $0.827 \pm 0.022 \mathrm{aA}$ \\
\hline 45 & 3.6 & $14.28 \pm 1.58 \mathrm{aA}$ & $1.17 \pm 0.05 \mathrm{bA}$ & $5.51 \pm 0.53 \mathrm{abA}$ & $0.809 \pm 0.023 \mathrm{abB}$ \\
\hline 30 & 3.6 & $13.43 \pm 1.16 \mathrm{aA}$ & $1.45 \pm 0.17 \mathrm{bA}$ & $4.41 \pm 0.70 \mathrm{bB}$ & $0.829 \pm 0.025 \mathrm{aA}$ \\
\hline 15 & 3.6 & $16.05 \pm 1.81 \mathrm{aAB}$ & $1.23 \pm 0.08 \mathrm{bA}$ & $5.10 \pm 0.05 \mathrm{ab} A B$ & $0.796 \pm 0.029 \mathrm{bB}$ \\
\hline 0 & 3.6 & $14.51 \pm 1.39 \mathrm{aA}$ & $2.24 \pm 0.48 \mathrm{aA}$ & $6.17 \pm 0.43 \mathrm{aA}$ & $0.816 \pm 0.022 \mathrm{abA}$ \\
\hline 45 & 5.4 & $15.75 \pm 1.35 \mathrm{aA}$ & $1.36 \pm 0.16 \mathrm{aA}$ & $5.20 \pm 0.28 \mathrm{abA}$ & $0.815 \pm 0.011 \mathrm{aAB}$ \\
\hline 30 & 5.4 & $14.55 \pm 1.68 \mathrm{aA}$ & $1.46 \pm 0.09 \mathrm{aA}$ & $4.82 \pm 0.15 \mathrm{abAB}$ & $0.824 \pm 0.021 \mathrm{aA}$ \\
\hline 15 & 5.4 & $15.56 \pm 1.58 \mathrm{aB}$ & $1.40 \pm 0.06 \mathrm{aA}$ & $3.86 \pm 0.41 \mathrm{bB}$ & $0.805 \pm 0.028 \mathrm{aAB}$ \\
\hline 0 & 5.4 & $15.34 \pm 1.71 \mathrm{aA}$ & $1.51 \pm 0.20 \mathrm{aB}$ & $5.58 \pm 0.55 \mathrm{aA}$ & $0.819 \pm 0.022 \mathrm{aA}$ \\
\hline 45 & 7.2 & $14.80 \pm 1.27 \mathrm{abA}$ & $1.30 \pm 0.17 \mathrm{aA}$ & $4.77 \pm 0.22 \mathrm{aA}$ & $0.837 \pm 0.020 \mathrm{aA}$ \\
\hline 30 & 7.2 & $14.09 \pm 1.63 \mathrm{bA}$ & $1.31 \pm 0.20 \mathrm{aA}$ & $4.64 \pm 0.29 \mathrm{aA}$ & $0.824 \pm 0.018 \mathrm{aA}$ \\
\hline 15 & 7.2 & $17.20 \pm 0.69 \mathrm{aAB}$ & $1.30 \pm 0.04 \mathrm{aA}$ & $3.84 \pm 0.53 \mathrm{aA}$ & $0.796 \pm 0.027 \mathrm{bB}$ \\
\hline 0 & 7.2 & $12.95 \pm 1.26 \mathrm{bA}$ & $1.53 \pm 0.22 \mathrm{aB}$ & $4.28 \pm 0.64 \mathrm{aA}$ & $0.832 \pm 0.025 \mathrm{aA}$ \\
\hline 45 & 9.0 & $15.53 \pm 1.67 \mathrm{bA}$ & $1.25 \pm 0.08 \mathrm{aA}$ & $5.57 \pm 0.50 \mathrm{aA}$ & $0.818 \pm 0.017 \mathrm{abAB}$ \\
\hline 30 & 9.0 & $14.46 \pm 1.55 \mathrm{bA}$ & $1.46 \pm 0.08 \mathrm{aA}$ & $5.37 \pm 0.25 \mathrm{aAB}$ & $0.823 \pm 0.020 \mathrm{abA}$ \\
\hline 15 & 9.0 & $18.50 \pm 0.78 \mathrm{aA}$ & $1.29 \pm 0.10 \mathrm{aA}$ & $5.62 \pm 0.59 \mathrm{aA}$ & $0.800 \pm 0.029 \mathrm{bB}$ \\
\hline 0 & 9.0 & $15.72 \pm 0.93 \mathrm{bA}$ & $1.15 \pm 0.08 \mathrm{aB}$ & $4.47 \pm 0.93 \mathrm{aB}$ & $0.834 \pm 0.027 \mathrm{aA}$ \\
\hline 45 & & $15.21 \pm 0.56 \mathrm{~b}$ & $1.29 \pm 0.05 \mathrm{~b}$ & $5.32 \pm 0.17 \mathrm{a}$ & $0.821 \pm 0.008 \mathrm{a}$ \\
\hline 30 & & $14.44 \pm 0.58 b$ & $1.41 \pm 0.06 \mathrm{ab}$ & $4.95 \pm 0.19 \mathrm{ab}$ & $0.826 \pm 0.009 \mathrm{a}$ \\
\hline 15 & & $16.60 \pm 0.55 \mathrm{a}$ & $1.32 \pm 0.04 \mathrm{~b}$ & $4.79 \pm 0.25 b$ & $0.805 \pm 0.011 \mathrm{~b}$ \\
\hline 0 & & $14.84 \pm 0.54 \mathrm{~b}$ & $1.56 \pm 0.13 \mathrm{a}$ & $5.21 \pm 0.29 \mathrm{ab}$ & $0.826 \pm 0.009 \mathrm{a}$ \\
\hline 0 & & $15.69 \pm 0.32 \mathrm{AB}$ & $1.37 \pm 0.06 \mathrm{~A}$ & $5.53 \pm 0.17 \mathrm{~A}$ & $0.827 \pm 0.010 \mathrm{~A}$ \\
\hline 3.6 & & $14.57 \pm 0.72 \mathrm{~B}$ & $1.52 \pm 0.16 \mathrm{~A}$ & $5.30 \pm 0.27 \mathrm{AB}$ & $0.812 \pm 0.011 \mathrm{~B}$ \\
\hline 5.4 & & $15.30 \pm 0.72 \mathrm{AB}$ & $1.43 \pm 0.06 \mathrm{~A}$ & $4.87 \pm 0.24 \mathrm{BC}$ & $0.816 \pm 0.010 \mathrm{AB}$ \\
\hline 7.2 & & $14.76 \pm 0.69 \mathrm{AB}$ & $1.36 \pm 0.08 \mathrm{~A}$ & $4.38 \pm 0.22 \mathrm{C}$ & $0.822 \pm 0.011 \mathrm{AB}$ \\
\hline 9.0 & & $16.05 \pm 0.69 \mathrm{~A}$ & $1.29 \pm 0.05 \mathrm{~A}$ & $5.26 \pm 0.30 \mathrm{AB}$ & $0.819 \pm 0.011 \mathrm{AB}$ \\
\hline \multicolumn{6}{|l|}{ Variation source } \\
\hline Application time (T) & & 0.0021 & 0.0555 & 0.0994 & $<0.001$ \\
\hline Rate (R) & & 0.1163 & 0.3592 & $<0.001$ & 0.0862 \\
\hline$T \times R$ & & 0.4352 & 0.0666 & 0.0125 & 0.284 \\
\hline Block & & $<0.001$ & 0.1971 & $<0.001$ & $<0.001$ \\
\hline $\mathrm{CV}(\%)$ & & 11.67 & 23.89 & 14.24 & 1.93 \\
\hline
\end{tabular}

${ }^{(1)}$ Means followed by equal letters, lowercase letters compare application times and uppercase letters compare the different rates applied, do not differ by Duncan's test, at $5 \%$ probability. ${ }^{(2)}$ Days before soybean sowing. CV, coefficient of variation. 
of thermal dissipation mechanisms was not sufficient to prevent changes in the electron transport and photosynthetic efficiency of soybean plants, as was also found for cucumber plants exposed to the residual effect of bensulfuron-methyl (Sun et al., 2019).

The yield components, in general, were more responsive to herbicide application in 2017/2018. In this crop year, the number of pods and number of grains were compromised at all application intervals from 0 to $45 \mathrm{DBS}$ and at the metsulfuron-methyl rates from 1.8 to $7.2 \mathrm{~g} \mathrm{ha}^{-1}$ (Figure $4 \mathrm{~A}$ and $\mathrm{C}$ and Table 4). In 2018/2019, an increase of 35 and 39\%, respectively, was observed in number of pods and number of grains between applications at 0 and 15 DBS for rates above $3.6 \mathrm{~g} \mathrm{ha}^{-1}$ (Figure $4 \mathrm{~B}$ and D and Table 4). The density of the plants was causally related to soybean yield

Table 3. Initial fluorescence $\left(\mathrm{F}_{0}\right)$, potential quantum yield of PSII $\left(\mathrm{F}_{\mathrm{v}} / \mathrm{F}_{\mathrm{m}}\right)$, electron transport rate (ETR), quenching photochemical (qP), photochemical extinction coefficient (qL), and nonphotochemical quenching (NPQ) of the NA 5909 RG soybean (Glycine max) cultivar sown 45, 30, 15, and 0 days after the application of metsulfuron-methyl at different rates $\left(0,3.6,5.4,7.2\right.$, and $\left.9.0 \mathrm{~g} \mathrm{ha}^{-1}\right)$, in the 2018/2019 crop year, in the municipality of Erechim, in the state of Rio Grande do Sul, Brazil $^{(1)}$.

\begin{tabular}{|c|c|c|c|c|c|c|c|}
\hline $\begin{array}{l}\text { Time } \\
(\mathrm{DBS})^{(2)}\end{array}$ & $\begin{array}{c}\text { Rate } \\
\text { (g ha-1 a.i.) }\end{array}$ & $\mathrm{F}_{0}$ & $\mathrm{~F}_{\mathrm{v}} / \mathrm{F}_{\mathrm{m}}$ & ETR & $\mathrm{qP}$ & $\mathrm{qL}$ & NPQ \\
\hline 45 & 0 & $163.7 \pm 5.3 \mathrm{aA}$ & $0.762 \pm 0.026 \mathrm{aA}$ & $44.2 \pm 11.7 \mathrm{aBC}$ & $0.925 \pm 0.131 \mathrm{aA}$ & $0.623 \pm 0.123 \mathrm{aA}$ & $1.62 \pm 0.09 \mathrm{aAB}$ \\
\hline 30 & 0 & $163.7 \pm 5.3 \mathrm{aA}$ & $0.762 \pm 0.026 \mathrm{aA}$ & $44.2 \pm 11.7 \mathrm{aA}$ & $0.925 \pm 0.131 \mathrm{aA}$ & $0.623 \pm 0.123 \mathrm{aA}$ & $1.62 \pm 0.09 \mathrm{aA}$ \\
\hline 15 & 0 & $163.7 \pm 5.3 \mathrm{aA}$ & $0.762 \pm 0.026 \mathrm{aA}$ & $44.2 \pm 11.7 \mathrm{aA}$ & $0.925 \pm 0.131 \mathrm{aA}$ & $0.623 \pm 0.123 \mathrm{aA}$ & $1.62 \pm 0.09 \mathrm{aA}$ \\
\hline 0 & 0 & $163.7 \pm 5.3 \mathrm{aA}$ & $0.762 \pm 0.026 \mathrm{aAB}$ & $44.2 \pm 11.7 \mathrm{aA}$ & $0.925 \pm 0.131 \mathrm{aA}$ & $0.623 \pm 0.123 \mathrm{aA}$ & $1.62 \pm 0.09 \mathrm{aAB}$ \\
\hline 45 & 3.6 & $145.0 \pm 8.6 \mathrm{bAC}$ & $0.790 \pm 0.016 \mathrm{aA}$ & $99.4 \pm 3.3 \mathrm{aA}$ & $0.898 \pm 0.180 \mathrm{aA}$ & $0.583 \pm 0.153 \mathrm{aA}$ & $1.68 \pm 0.24 \mathrm{abAB}$ \\
\hline 30 & 3.6 & $171.0 \pm 9.4 \mathrm{aA}$ & $0.756 \pm 0.027 \mathrm{bA}$ & $35.6 \pm 6.8 \mathrm{bA}$ & $0.840 \pm 0.197 \mathrm{aA}$ & $0.528 \pm 0.148 \mathrm{aA}$ & $2.08 \pm 0.41 \mathrm{aA}$ \\
\hline 15 & 3.6 & $117.7 \pm 5.4 \mathrm{cC}$ & $0.772 \pm 0.028 \mathrm{abA}$ & $46.8 \pm 14.8 \mathrm{bA}$ & $0.626 \pm 0.042 \mathrm{aA}$ & $0.352 \pm 0.036 \mathrm{aA}$ & $1.62 \pm 0.09 \mathrm{bA}$ \\
\hline 0 & 3.6 & $147.3 \pm 8.6 \mathrm{bAB}$ & $0.778 \pm 0.024 \mathrm{abA}$ & $33.5 \pm 11.4 \mathrm{bA}$ & $0.817 \pm 0.097 \mathrm{aA}$ & $0.527 \pm 0.090 \mathrm{aA}$ & $2.18 \pm 0.34 \mathrm{aA}$ \\
\hline 45 & 5.4 & $137.0 \pm 14.8 \mathrm{aBC}$ & $0.784 \pm 0.028 \mathrm{aA}$ & $56.2 \pm 15.7 \mathrm{aB}$ & $0.758 \pm 0.103 \mathrm{aA}$ & $0.468 \pm 0.094 \mathrm{aA}$ & $2.18 \pm 0.07 \mathrm{aA}$ \\
\hline 30 & 5.4 & $121.0 \pm 5.0 \mathrm{aB}$ & $0.765 \pm 0.034 \mathrm{aA}$ & $39.0 \pm 10.3 \mathrm{aA}$ & $0.674 \pm 0.014 \mathrm{aA}$ & $0.393 \pm 0.009 \mathrm{aA}$ & $1.90 \pm 0.30 \mathrm{aA}$ \\
\hline 15 & 5.4 & $116.7 \pm 2.3 \mathrm{aC}$ & $0.785 \pm 0.016 \mathrm{aA}$ & $47.0 \pm 17.5 \mathrm{aA}$ & $0.720 \pm 0.024 \mathrm{aA}$ & $0.432 \pm 0.026 \mathrm{aA}$ & $1.35 \pm 0.07 \mathrm{bA}$ \\
\hline 0 & 5.4 & $126.5 \pm 7.3 \mathrm{aBC}$ & $0.775 \pm 0.019 \mathrm{aA}$ & $35.2 \pm 10.6 \mathrm{aA}$ & $0.721 \pm 0.028 \mathrm{aA}$ & $0.439 \pm 0.024 \mathrm{aA}$ & $1.73 \pm 0.24 \mathrm{abAB}$ \\
\hline 45 & 7.2 & $161.3 \pm 9.9 \mathrm{aAB}$ & $0.786 \pm 0.023 \mathrm{aA}$ & $50.0 \pm 16.3 \mathrm{aBC}$ & $0.623 \pm 0.153 \mathrm{aA}$ & $0.388 \pm 0.123 \mathrm{aA}$ & $2.00 \pm 0.06 \mathrm{aA}$ \\
\hline 30 & 7.2 & $160.7 \pm 12.7 \mathrm{aA}$ & $0.785 \pm 0.018 \mathrm{aA}$ & $46.1 \pm 11.0 \mathrm{abA}$ & $0.869 \pm 0.106 \mathrm{aA}$ & $0.577 \pm 0.107 \mathrm{aA}$ & $1.82 \pm 0.28 \mathrm{aA}$ \\
\hline 15 & 7.2 & $116.3 \pm 1.3 \mathrm{bBC}$ & $0.795 \pm 0.013 \mathrm{aA}$ & $29.2 \pm 6.7 \mathrm{abA}$ & $0.638 \pm 0.028 \mathrm{aA}$ & $0.359 \pm 0.022 \mathrm{aA}$ & $1.55 \pm 0.05 \mathrm{aA}$ \\
\hline 0 & 7.2 & $111.0 \pm 1.5 \mathrm{bC}$ & $0.783 \pm 0.029 \mathrm{aA}$ & $23.9 \pm 1.6 \mathrm{bA}$ & $0.653 \pm 0.047 \mathrm{aA}$ & $0.375 \pm 0.036 \mathrm{aA}$ & $1.61 \pm 0.03 \mathrm{aAB}$ \\
\hline 45 & 9.0 & $127.7 \pm 11.4 \mathrm{bC}$ & $0.765 \pm 0.024 \mathrm{aA}$ & $26.0 \pm 11.9 \mathrm{abC}$ & $0.636 \pm 0.083 \mathrm{aA}$ & $0.390 \pm 0.074 \mathrm{aA}$ & $1.22 \pm 0.45 \mathrm{aB}$ \\
\hline 30 & 9.0 & $160.0 \pm 9.2 \mathrm{aA}$ & $0.766 \pm 0.026 \mathrm{aA}$ & $39.8 \pm 12.7 \mathrm{abA}$ & $0.731 \pm 0.122 \mathrm{aA}$ & $0.457 \pm 0.102 \mathrm{aA}$ & $1.79 \pm 0.22 \mathrm{aA}$ \\
\hline 15 & 9.0 & $141.3 \pm 14.3 \mathrm{abAB}$ & $0.772 \pm 0.016 \mathrm{aA}$ & $46.9 \pm 17.9 \mathrm{aA}$ & $0.777 \pm 0.165 \mathrm{aA}$ & $0.493 \pm 0.140 \mathrm{aA}$ & $1.76 \pm 0.07 \mathrm{aA}$ \\
\hline 0 & 9.0 & $128.3 \pm 10.9 \mathrm{bBC}$ & $0.742 \pm 0.034 \mathrm{aB}$ & $19.6 \pm 0.1 \mathrm{bA}$ & $0.717 \pm 0.016 \mathrm{aA}$ & $0.432 \pm 0.012 \mathrm{aA}$ & $1.56 \pm 0.02 \mathrm{aB}$ \\
\hline 45 & & $146.9 \pm 5.2 \mathrm{a}$ & $0.778 \pm 0.010 \mathrm{a}$ & $55.2 \pm 7.52 \mathrm{a}$ & $0.768 \pm 0.061 \mathrm{a}$ & $0.490 \pm 0.051 \mathrm{a}$ & $1.74 \pm 0.12 \mathrm{ab}$ \\
\hline 30 & & $155.3 \pm 5.3 \mathrm{a}$ & $0.767 \pm 0.011 \mathrm{a}$ & $40.9 \pm 4.34 b c$ & $0.808 \pm 0.055 \mathrm{a}$ & $0.516 \pm 0.047 \mathrm{a}$ & $1.84 \pm 0.12 \mathrm{a}$ \\
\hline 15 & & $131.1 \pm 5.2 b$ & $0.777 \pm 0.009 a$ & $42.8 \pm 5.92 b$ & $0.737 \pm 0.046 \mathrm{a}$ & $0.452 \pm 0.041 \mathrm{a}$ & $1.51 \pm 0.05 b$ \\
\hline 0 & & $135.4 \pm 5.0 \mathrm{~b}$ & $0.768 \pm 0.011 \mathrm{a}$ & $31.3 \pm 3.91 \mathrm{c}$ & $0.767 \pm 0.037 \mathrm{a}$ & $0.479 \pm 0.034 \mathrm{a}$ & $1.74 \pm 0.09 \mathrm{ab}$ \\
\hline 0 & & $163.7 \pm 2.4 \mathrm{~A}$ & $0.762 \pm 0.012 \mathrm{~B}$ & $44.2 \pm 5.24 \mathrm{AB}$ & $0.925 \pm 0.059 \mathrm{~A}$ & $0.623 \pm 0.055 \mathrm{~A}$ & $1.62 \pm 0.04 \mathrm{~A}$ \\
\hline 3.6 & & $145.3 \pm 6.1 \mathrm{~B}$ & $0.774 \pm 0.011 \mathrm{AB}$ & $53.8 \pm 8.27 \mathrm{~A}$ & $0.795 \pm 0.069 \mathrm{AB}$ & $0.497 \pm 0.057 \mathrm{~B}$ & $1.82 \pm 0.16 \mathrm{~A}$ \\
\hline 5.4 & & $125.3 \pm 4.4 \mathrm{C}$ & $0.777 \pm 0.012 \mathrm{AB}$ & $44.4 \pm 6.55 \mathrm{AB}$ & $0.718 \pm 0.026 \mathrm{~B}$ & $0.433 \pm 0.024 \mathrm{~B}$ & $1.77 \pm 0.12 \mathrm{~A}$ \\
\hline 7.2 & & $137.3 \pm 7.1 \mathrm{~B}$ & $0.787 \pm 0.010 \mathrm{~A}$ & $37.3 \pm 5.46 \mathrm{~B}$ & $0.696 \pm 0.051 \mathrm{~B}$ & $0.425 \pm 0.044 \mathrm{~B}$ & $1.75 \pm 0.08 \mathrm{~A}$ \\
\hline 9.0 & & $139.3 \pm 6.2 \mathrm{~B}$ & $0.761 \pm 0.012 \mathrm{~B}$ & $33.1 \pm 6.25 \mathrm{~B}$ & $0.715 \pm 0.051 \mathrm{~B}$ & $0.443 \pm 0.043 \mathrm{~B}$ & $1.58 \pm 0.13 \mathrm{~A}$ \\
\hline \multicolumn{8}{|c|}{ Variation source } \\
\hline \multicolumn{2}{|c|}{ Application time (T) } & $<0.001$ & 0.2599 & $<0.001$ & 0.7449 & 0.7091 & 0.0514 \\
\hline \multicolumn{2}{|l|}{ Rate (R) } & $<0.001$ & 0.0073 & 0.0106 & 0.0122 & 0.011 & 0.3375 \\
\hline \multicolumn{2}{|l|}{$\mathrm{T} \times \mathrm{R}$} & $<0.001$ & $<0.001$ & 0.0016 & 0.7621 & 0.7846 & 0.0314 \\
\hline \multicolumn{2}{|l|}{ Block } & 0.0419 & 0.7684 & $<0.001$ & 0.0021 & 0.0013 & 0.0017 \\
\hline \multicolumn{2}{|l|}{ CV (\%) } & 11.54 & 2.85 & 39.09 & 26.21 & 35.95 & 22.08 \\
\hline
\end{tabular}

(1) Means followed by equal letters, lowercase letters compare application times and uppercase letters compare the different rates applied, do not differ by Duncan's test, at $5 \%$ probability. ${ }^{(2)}$ Days before soybean sowing. CV, coefficient of variation. 

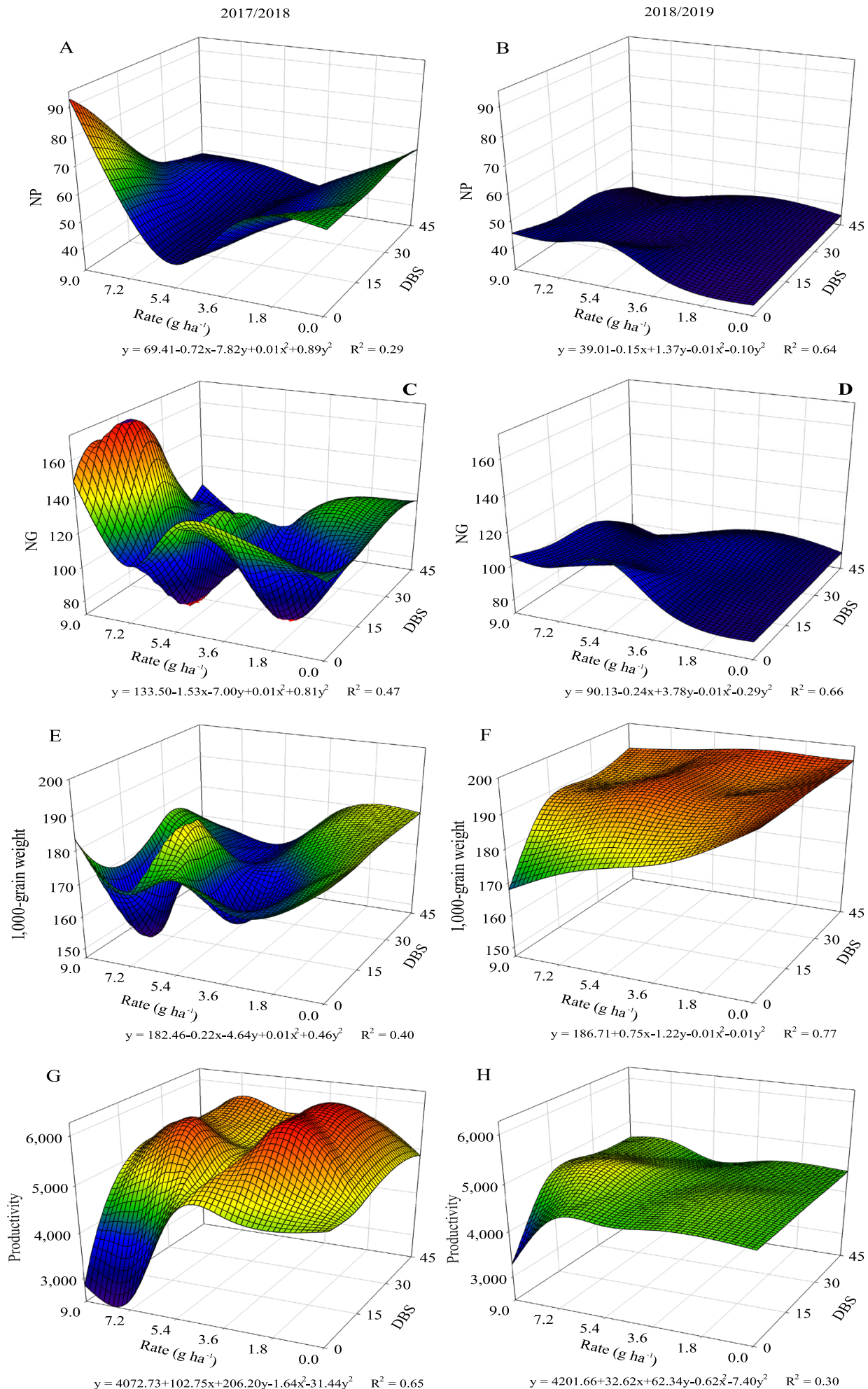

Figure 4. Number of pods (NP) (A and B), number of grains (NG) (C and D), 1,000-grain weight (g) (E and F), and productivity $\left(\mathrm{kg} \mathrm{ha}^{-1}\right)(\mathrm{G}$ and $\mathrm{H})$ of the NA 5909 RG soybean (Glycine max) cultivar sown 45, 30, 15, and 0 days after the application of metsulfuron-methyl at different rates $\left(0,3.6,5.4,7.2\right.$, and $\left.9.0 \mathrm{~g} \mathrm{ha}^{-1}\right)$, in two consecutive crop years - 2017/2018 (A, C, E, and G) and 2018/2019 (B, D, F, and H) - in the municipality of Erechim, in the state of Rio Grande do Sul, Brazil. DBS, days before soybean sowing. 
components. As mentioned before, the application of metsulfuron-methyl at 0 DBS caused high plant mortality, changing the density of the plants by area. This reduction in plant population stimulated the phenotypic plasticity of soybean plants, a phenomenon that has been reported to allow plants to change their morphology and regulate their growth and production components according to different densities, emitting more branches and increasing branch growth at low densities (Ferreira et al., 2016; Balbinot Junior et al., 2018). The authors concluded that number of pods was the main variable associated with phenotypic plasticity, which, in the present study, explains its significant increase in the second crop year, mainly at 0 DBS (Table 4).

Conversely, the 1,000-grain weight showed lower values with the increase of the metsulfuron-methyl rate and proximity to soybean sowing (Figure $4 \mathrm{E}$ and

Table 4. Number of pods (NP), number of grains (NG), 1,000-grain weight, and productivity of the NA 5909 RG soybean (Glycine max) cultivar sown 45, 30, 15, and 0 days after the application of metsulfuron-methyl at different rates $(0,3.6,5.4$, 7.2, and $9.0 \mathrm{~g} \mathrm{ha}^{-1}$ ), in two consecutive crop years (2017/2018 and 2018/2019), in the municipality of Erechim, in the state of Rio Grande do Sul, Brazil(1).

\begin{tabular}{|c|c|c|c|c|c|c|c|c|c|}
\hline \multicolumn{2}{|l|}{ Factor } & \multicolumn{4}{|c|}{ 2017/2018 crop year } & \multicolumn{4}{|c|}{ 2018/2019 crop year } \\
\hline $\begin{array}{l}\text { Time } \\
(\mathrm{DBS})^{(2)} \\
\end{array}$ & $\begin{array}{c}\text { Rate } \\
\left(\mathrm{g} \mathrm{ha}^{-1} \text { a.i. }\right) \\
\end{array}$ & NP & $\mathrm{NG}$ & $\begin{array}{c}1,000 \text {-grain } \\
\text { weight }(\mathrm{g})\end{array}$ & $\begin{array}{c}\text { Productivity } \\
\left(\mathrm{kg} \mathrm{ha}^{-1}\right)\end{array}$ & NP & NG & $\begin{array}{c}\text { 1,000-grain } \\
\text { weight }(\mathrm{g})\end{array}$ & $\begin{array}{c}\text { Productivity } \\
\left(\mathrm{kg} \mathrm{ha}^{-1}\right)\end{array}$ \\
\hline 45 & 0 & $62.1 \pm 3.4 \mathrm{aA}$ & $115.9 \pm 16.8 \mathrm{aA}$ & $180.2 \pm 6.0 \mathrm{aA}$ & $4857.2 \pm 158.4 \mathrm{aB}$ & $35.4 \pm 3.3 \mathrm{aA}$ & $81.9 \pm 9.0 \mathrm{aA}$ & $195.78 \pm 1.0 \mathrm{aA}$ & $4468.0 \pm 81.2 \mathrm{aA}$ \\
\hline 30 & 0 & $62.1 \pm 3.4 \mathrm{aA}$ & $115.9 \pm 16.8 \mathrm{aA}$ & $180.2 \pm 6.0 \mathrm{aA}$ & $4857.2 \pm 158.4 \mathrm{aB}$ & $35.4 \pm 3.3 \mathrm{aA}$ & $81.9 \pm 9.0 \mathrm{aA}$ & $195.78 \pm 1.0 \mathrm{aA}$ & $4468.0 \pm 81.2 \mathrm{aA}$ \\
\hline 15 & 0 & $62.1 \pm 3.4 \mathrm{aA}$ & $115.9 \pm 16.8 \mathrm{aAB}$ & $180.2 \pm 6.0 \mathrm{aAB}$ & $4857.2 \pm 158.4 \mathrm{aB}$ & $35.4 \pm 3.3 \mathrm{aA}$ & $81.9 \pm 9.0 \mathrm{aA}$ & $195.78 \pm 1.0 \mathrm{aA}$ & $4468.0 \pm 81.2 \mathrm{aA}$ \\
\hline 0 & 0 & $62.1 \pm 3.4 \mathrm{aB}$ & $115.9 \pm 16.8 \mathrm{aA}$ & $180.2 \pm 6.0 \mathrm{aA}$ & $4857.2 \pm 158.4 \mathrm{aA}$ & $35.4 \pm 3.3 \mathrm{aC}$ & $81.9 \pm 9.0 \mathrm{aC}$ & $195.78 \pm 1.0 \mathrm{aA}$ & $4468.0 \pm 81.2 \mathrm{aA}$ \\
\hline 45 & 3.6 & $42.1 \pm 3.9 \mathrm{aB}$ & $94.1 \pm 2.7 \mathrm{abA}$ & $175.1 \pm 3.6 \mathrm{aA}$ & $5662.3 \pm 291.5 \mathrm{aA}$ & $37.9 \pm 1.5 \mathrm{aA}$ & $88.0 \pm 3.6 \mathrm{aA}$ & $196.0 \pm 3.4 \mathrm{aA}$ & $4428.7 \pm 120.0 \mathrm{aA}$ \\
\hline 30 & 3.6 & $47.8 \pm 2.2 \mathrm{aAB}$ & $109.5 \pm 3.3 \mathrm{abA}$ & $171.1 \pm 6.1 \mathrm{aA}$ & $5920.6 \pm 313.3 \mathrm{aA}$ & $40.4 \pm 1.5 \mathrm{aA}$ & $96.1 \pm 3.3 \mathrm{aA}$ & $191.4 \pm 4.4 \mathrm{aA}$ & $4525.2 \pm 60.6 \mathrm{aA}$ \\
\hline 15 & 3.6 & $42.6 \pm 2.0 \mathrm{aB}$ & $72.3 \pm 9.2 \mathrm{bB}$ & $182.3 \pm 6.9 \mathrm{aA}$ & $5698.1 \pm 145.1 \mathrm{aA}$ & $39.0 \pm 2.5 \mathrm{aA}$ & $92.5 \pm 5.0 \mathrm{aA}$ & $191.3 \pm 6.9 \mathrm{aA}$ & $4640.2 \pm 70.5 \mathrm{aAB}$ \\
\hline 0 & 3.6 & $54.7 \pm 11.0 \mathrm{aB}$ & $134.7 \pm 25.0 \mathrm{aA}$ & $169.3 \pm 4.2 \mathrm{aA}$ & $4825.5 \pm 229.7 \mathrm{bA}$ & $40.2 \pm 2.2 \mathrm{aBC}$ & $95.0 \pm 3.8 \mathrm{aBC}$ & $181.8 \pm 3.8 \mathrm{bB}$ & $4527.1 \pm 104.4 \mathrm{aA}$ \\
\hline 45 & 5.4 & $45.6 \pm 1.1 \mathrm{aB}$ & $80.4 \pm 4.0 .0 \mathrm{bA}$ & $164.3 \pm 5.1 \mathrm{aA}$ & $5474.5 \pm 51.9 \mathrm{aAB}$ & $35.3 \pm 1.5 \mathrm{bA}$ & $82.7 \pm 1.3 \mathrm{bA}$ & $194.7 \pm 4.2 \mathrm{aA}$ & $4310.4 \pm 127.6 \mathrm{aA}$ \\
\hline 30 & 5.4 & $41.6 \pm 3.9 \mathrm{aB}$ & $80.3 \pm 3.8 \mathrm{bA}$ & $165.1 \pm 4.0 \mathrm{aA}$ & $5099.3 \pm 67.2 \mathrm{abB}$ & $39.9 \pm 4.8 \mathrm{bA}$ & $94.0 \pm 12.0 \mathrm{bA}$ & $193.6 \pm 4.9 \mathrm{aA}$ & $4606.4 \pm 89.7 \mathrm{aA}$ \\
\hline 15 & 5.4 & $45.3 \pm 6.1 \mathrm{aB}$ & $86.9 \pm 9.1 \mathrm{bAB}$ & $159.9 \pm 1.5 \mathrm{aC}$ & $5868.1 \pm 24.2 \mathrm{aA}$ & $37.93 \pm 1.4 \mathrm{bA}$ & $94.8 \pm 8.1 \mathrm{bA}$ & $193.0 \pm 1.6 \mathrm{aA}$ & $4602.2 \pm 34.7 \mathrm{aAB}$ \\
\hline 0 & 5.4 & $40.3 \pm 5.8 \mathrm{aC}$ & $131.8 \pm 39.9 \mathrm{aA}$ & $175.7 \pm 6.8 \mathrm{aA}$ & $4700.9 \pm 420.4 \mathrm{bA}$ & $47.9 \pm 1.9 \mathrm{aA}$ & $113.4 \pm 3.6 \mathrm{aA}$ & $178.7 \pm 1.2 \mathrm{bB}$ & $4455.3 \pm 289.6 \mathrm{aA}$ \\
\hline 45 & 7.2 & $48.1 \pm 4.0 \mathrm{abAB}$ & $87.9 \pm 8.9 \mathrm{aA}$ & $165.6 \pm 4.0 \mathrm{aA}$ & $5730.1 \pm 204.2 \mathrm{aA}$ & $32.3 \pm 0.6 \mathrm{bA}$ & $73.9 \pm 2.2 \mathrm{bA}$ & $193.1 \pm 1.1 \mathrm{aA}$ & $4644.9 \pm 77.7 \mathrm{abA}$ \\
\hline 30 & 7.2 & $53.1 \pm 2.8 \mathrm{abAB}$ & $107.9 \pm 0.6 \mathrm{aA}$ & $169.6 \pm 5.3 \mathrm{aA}$ & $5498.9 \pm 168.2 \mathrm{aAB}$ & $38.8 \pm 1.1 \mathrm{abA}$ & $90.7 \pm 2.1 \mathrm{aA}$ & $188.9 \pm 1.2 \mathrm{aA}$ & $4721.4 \pm 120.3 \mathrm{abA}$ \\
\hline 15 & 7.2 & $40.7 \pm 4.2 \mathrm{bB}$ & $110.1 \pm 4.6 \mathrm{aAB}$ & $166.6 \pm 2.0 \mathrm{aBC}$ & $5118.0 \pm 62.5 \mathrm{aAB}$ & $41.4 \pm 2.9 \mathrm{aA}$ & $93.9 \pm 5.9 \mathrm{aA}$ & $189.9 \pm 2.6 \mathrm{aA}$ & $4970.6 \pm 84.4 \mathrm{aA}$ \\
\hline 0 & 7.2 & $57.5 \pm 7.7 \mathrm{aB}$ & $105.6 \pm 12.8 \mathrm{aA}$ & $169.2 \pm 7.0 \mathrm{aA}$ & $2678.2 \pm 305.0 \mathrm{bB}$ & $45.2 \pm 1.4 \mathrm{aAB}$ & $104.1 \pm 4.7 \mathrm{aAB}$ & $174.5 \pm 4.4 \mathrm{bBC}$ & $4430.6 \pm 277.1 \mathrm{aA}$ \\
\hline 45 & 9.0 & $48.5 \pm 5.3 \mathrm{bAB}$ & $107.4 \pm 4.6 \mathrm{abA}$ & $169.1 \pm 5.9 \mathrm{abA}$ & $4988.0 \pm 223.9 \mathrm{aAB}$ & $33.6 \pm 3.6 \mathrm{bA}$ & $79.3 \pm 8.4 \mathrm{bA}$ & $192.1 \pm 4.4 \mathrm{aA}$ & $4620.3 \pm 165.8 \mathrm{aA}$ \\
\hline 30 & 9.0 & $45.4 \pm 1.7 \mathrm{bB}$ & $94.3 \pm 5.5 \mathrm{bA}$ & $171.7 \pm 7.2 \mathrm{abA}$ & $5515.1 \pm 76.9 \mathrm{aAB}$ & $40.5 \pm 1.6 \mathrm{abA}$ & $95.9 \pm 3.0 \mathrm{abA}$ & $188.5 \pm 3.0 \mathrm{aA}$ & $4674.7 \pm 92.0 \mathrm{aA}$ \\
\hline 15 & 9.0 & $52.7 \pm 5.8 \mathrm{bAB}$ & $127.2 \pm 3.0 \mathrm{abA}$ & $159.8 \pm 6.1 \mathrm{bC}$ & $5671.6 \pm 253.0 \mathrm{aA}$ & $40.9 \pm 2.3 \mathrm{abA}$ & $98.2 \pm 5.1 \mathrm{aA}$ & $188.3 \pm 4.6 \mathrm{aA}$ & $4743.6 \pm 65.0 \mathrm{aAB}$ \\
\hline 0 & 9.0 & $92.7 \pm 3.0 \mathrm{aA}$ & $148.7 \pm 9.2 \mathrm{aA}$ & $183.3 \pm 9.3 \mathrm{aA}$ & $2832.2 \pm 673.5 \mathrm{bB}$ & $45.4 \pm 1.4 \mathrm{aAB}$ & $105.7 \pm 3.0 \mathrm{aAB}$ & $168.0 \pm 4.0 \mathrm{bC}$ & $3274.3 \pm 344.1 \mathrm{bB}$ \\
\hline & 45 & $49.3 \pm 2.1 b$ & $97.1 \pm 4.7 b$ & $170.8 \pm 2.5 \mathrm{a}$ & $5342.4 \pm 116.7 \mathrm{a}$ & $34.9 \pm 1.1 \mathrm{c}$ & $81.2 \pm 2.6 \mathrm{c}$ & $194.5 \pm 1.2 \mathrm{a}$ & $4494.5 \pm 54.8 \mathrm{a}$ \\
\hline & 30 & $50.0 \pm 2.0 \mathrm{~b}$ & $101.6 \pm 4.4 \mathrm{~b}$ & $171.5 \pm 2.6 \mathrm{a}$ & $5378.2 \pm 110.6 \mathrm{a}$ & $39.0 \pm 1.2 \mathrm{~b}$ & $91.7 \pm 3.1 \mathrm{~b}$ & $191.6 \pm 1.5 \mathrm{a}$ & $4599.1 \pm 41.9 \mathrm{a}$ \\
\hline & 15 & $48.7 \pm 2.6 b$ & $102.5 \pm 6.0 \mathrm{~b}$ & $169.8 \pm 3.0 \mathrm{a}$ & $5442.6 \pm 107.2 \mathrm{a}$ & $38.9 \pm 1.1 \mathrm{~b}$ & $92.2 \pm 3.0 \mathrm{~b}$ & $191.7 \pm 1.7 \mathrm{a}$ & $4684.9 \pm 47.4 \mathrm{a}$ \\
\hline & 0 & $61.4 \pm 4.8 \mathrm{a}$ & $127.3 \pm 9.9 \mathrm{a}$ & $175.5 \pm 3.0 \mathrm{a}$ & $3978.8 \pm 279.5 b$ & $42.8 \pm 1.3 \mathrm{a}$ & $100.0 \pm 3.2 \mathrm{a}$ & $179.7 \pm 2.5 \mathrm{~b}$ & $4231.1 \pm 146.5 b$ \\
\hline & 0 & $62.1 \pm 1.5 \mathrm{~A}$ & $115.9 \pm 7.5 \mathrm{AB}$ & $180.2 \pm 2.7 \mathrm{~A}$ & $4857.2 \pm 70.8 \mathrm{~B}$ & $35.4 \pm 1.5 \mathrm{~B}$ & $81.9 \pm 4.0 \mathrm{~B}$ & $196.0 \pm 0.4 \mathrm{~A}$ & $4468.0 \pm 36.3 \mathrm{~B}$ \\
\hline & 3.6 & $46.8 \pm 3.0 \mathrm{~B}$ & $102.6 \pm 8.4 \mathrm{AB}$ & $174.5 \pm 2.7 \mathrm{AB}$ & $5526.6 \pm 156.3 \mathrm{~A}$ & $39.4 \pm 0.9 \mathrm{~A}$ & $92.9 \pm 1.9 \mathrm{~A}$ & $190.1 \pm 2.5 \mathrm{~B}$ & $4530.3 \pm 45.5 \mathrm{AB}$ \\
\hline & 5.4 & $43.2 \pm 2.2 \mathrm{~B}$ & $94.9 \pm 10.8 \mathrm{~B}$ & $166.2 \pm 2.6 \mathrm{C}$ & $5285.7 \pm 147.5 \mathrm{~A}$ & $40.3 \pm 1.7 \mathrm{~A}$ & $96.2 \pm 4.4 \mathrm{~A}$ & $190.0 \pm 2.3 \mathrm{~B}$ & $4493.6 \pm 80.4 \mathrm{AB}$ \\
\hline & 7.2 & $49.8 \pm 2.8 \mathrm{~B}$ & $102.9 \pm 4.3 \mathrm{AB}$ & $167.7 \pm 2.25 \mathrm{BC}$ & $4756.3 \pm 327.9 \mathrm{~B}$ & $39.4 \pm 1.4 \mathrm{~A}$ & $90.6 \pm 3.3 \mathrm{~A}$ & $186.6 \pm 2.2 \mathrm{BC}$ & $4691.9 \pm 87.8 \mathrm{~A}$ \\
\hline & 9.0 & $59.8 \pm 5.3 \mathrm{~A}$ & $119.4 \pm 6.0 \mathrm{~A}$ & $171.0 \pm 3.9 \mathrm{BC}$ & $4751.7 \pm 338.9 \mathrm{~B}$ & $40.1 \pm 1.5 \mathrm{~A}$ & $94.8 \pm 3.5 \mathrm{~A}$ & $184.2 \pm 3.0 \mathrm{C}$ & $4328.2 \pm 181.0 \mathrm{~B}$ \\
\hline \multicolumn{10}{|c|}{ Variation source } \\
\hline Applicati & ion time $(\mathrm{T})$ & $<0.001$ & 0.006 & 0.3364 & $<0.001$ & $<0.001$ & $<0.001$ & $<0.001$ & $<0.001$ \\
\hline Rate (R) & & $<0.001$ & 0.0996 & 0.0029 & $<0.001$ & 0.0443 & 0.0095 & $<0.001$ & 0.0186 \\
\hline $\mathrm{T} \times \mathrm{R}$ & & $<0.001$ & 0.2582 & 0.2418 & $<0.001$ & 0.2447 & 0.36 & 0.0102 & $<0.001$ \\
\hline Block & & 0.6977 & 0.6617 & 0.0038 & 0.8991 & 0.2589 & 0.0131 & $<0.001$ & 0.2345 \\
\hline CV (\%) & & 18.45 & 26.62 & 6.10 & 10.27 & 12.69 & 12.90 & 2.71 & 6.46 \\
\hline
\end{tabular}

${ }^{(1)}$ Means followed by equal letters, lowercase letters compare application times and uppercase letters compare the different rates applied, do not differ by Duncan's test, at $5 \%$ probability. ${ }^{(2)}$ Days before soybean sowing. CV, coefficient of variation. 
F and Table 4). In 2018/2919, the lowest 1,000-grain weight was observed with the application of rates above $3.6 \mathrm{~g} \mathrm{ha}^{-1}$, at 0 and $15 \mathrm{DBS}$ (Figure $4 \mathrm{~F}$ and Table 4). In both crop years, productivity was reduced mainly due to the highest rates of metsulfuron-methyl and the shorter interval between its application and soybean sowing, which was attributed to the residual effects of the herbicide in the soil. In 2017/2018, there was a reduction of up to $47 \%$ in productivity from 15 DBS onwards (Figure $4 \mathrm{G}$ and Table 4), whereas, in the following crop year, there was a $42 \%$ reduction when the highest rate was applied at 0 DBS (Figure $4 \mathrm{H}$ and Table 4). These yield components were also reported to be reduced in soybean plants after exposure to carryover of atrazine, metribuzin, flumioxazin, and a mixture of diuron, hexazinone, and sulfometuronmethyl (Gonçalves et al., 2018). The 1,000-grain weight is sensitive to various stress conditions, which directly affect crop yield (Souza et al., 2015; Comin et al., 2018), and may be greater with a lower number of pods per plant, which reduces competition for photoassimilates (Cruz et al., 2016).

As observed for the phytotoxicity data, there was a reduction in productivity in the shortest interval between herbicide application and soybean sowing (Figure 4 and Table 4). A decrease in yield was also found in soybean plants exposed to the residual effects of carotenoid synthesis- and photosystem-inhibitor herbicides after 42 days of application (Soltani et al., 2011). According to these authors, different herbicides caused injuries from $35 \%$ (isoxaflutole and atrazine) to 90\% (dimethenamid and dicamba/atrazine) on soybean plants grown afterwards, with a consequent reduction in biomass and crop yield. Therefore, to determine the necessary withdrawal period before sowing soybean, it is necessary to verify the interaction of the herbicide with the soil, whose particles may degrade or absorb it. These processes can last only a few days or even months or years in the case of highly persistent compounds (Filizola et al., 2002). This shows the importance of studies on the residual effects of different herbicides, in order to allow the selection of products less harmful to subsequent crops.

Since metsulfuron-methyl is applied at very low rates, its detection in the soil is difficult. This shows the need of using methods to determine the presence of its compounds in the soil (Marinho et al., 2019), in order to avoid sowing sensitive crops in the same area.
Among the methods used to identify soils contaminated by herbicides, bioindicator plants stand out as being quick and inexpensive, with cucumber being one of the most sensitive species to metsulfuron-methyl (Ismail \& Azlizan, 2002).

A moderate increase of $\sim 5 \%$ was observed in the phytotoxicity rates of cucumber plants grown after the application of the metsulfuron-methyl herbicide (Table 5). Moreover, there was an interaction between the rate and application interval of metsulfuronmethyl for initial fluorescence and nonphotochemical quenching. The herbicide promoted an increase in initial fluorescence and nonphotochemical quenching of up to 38 and $265 \%$ at 15 and 0 DBS, respectively. Initial fluorescence, like nonphotochemical quenching, is activated in adverse situations, specifically by a mechanism that involves the dissipation of excess energy via fluorescence in the antenna complex (Baker \& Rosenqvist, 2004). In cucumber plants, the increase in initial fluorescence and nonphotochemical quenching was probably sufficient to mitigate the negative effects of metsulfuron-methyl on the photosynthetic process, since the potential quantum yield of PSII $\left(\mathrm{F}_{\mathrm{v}} / \mathrm{F}_{\mathrm{m}}\right)$ and ETR were not altered.

However, unlike instantaneous physiological measurements, plant dry matter represents the accumulated effect of the metsulfuron-methyl residue in the soil. The shoot dry matter of cucumber plants showed a linear reduction of up to $18 \%$ with an increasing herbicide rate. A similar response was observed for the application intervals, with a greater reduction in shoot dry matter closer to cucumber sowing (Table 5). Similarly, residual bensulfuronmethyl affected the morphological traits of cucumber plants (Sun et al., 2019).

For the NA 5909 RG soybean cultivar, even though there were no major variations in productivity in the longest interval, the data set considering both experiments in the field does not allow a recommendation for safe sowing in a period equal to or less than 45 days after the application of metsulfuron-methyl. It has been suggested that the soybean crop should be sown at least 60 days after metsulfuron-methyl application since uncontrollable factors, such as environmental conditions, contribute to the process of herbicide persistence and degradation. 
Table 5. Phytotoxicity at 7 and 14 days after emergence (DAE), initial fluorescence $\left(\mathrm{F}_{0}\right)$, potential quantum yield of PSII $\left(\mathrm{F}_{\mathrm{v}} / \mathrm{F}_{\mathrm{m}}\right)$, electron transport rate (ETR), and nonphotochemical quenching (NPQ) of the Pioneiro cucumber (Cucumis sativus) cultivar sown 45, 30, 15, and 0 days after the application of metsulfuron-methyl at different rates $(0,3.6,5.4,7.2$, and 9.0 $\left.\mathrm{g} \mathrm{ha}^{-1}\right)$ in the municipality of Erechim, in the state of Rio Grande do Sul, Brazil ${ }^{(1)}$.

\begin{tabular}{|c|c|c|c|c|c|c|c|c|}
\hline \multicolumn{2}{|l|}{ Factor } & \multicolumn{2}{|c|}{ Phytotoxicity (\%) } & \multicolumn{4}{|c|}{ Chlorophylla $A$ fluorescence } & \multirow[b]{2}{*}{$\begin{array}{c}\mathrm{SDM}^{(3)} \\
(\mathrm{g})\end{array}$} \\
\hline $\begin{array}{l}\text { Time } \\
(\mathrm{DBS})^{(2)}\end{array}$ & $\begin{array}{c}\text { Rate } \\
\left(\mathrm{g} \mathrm{ha}^{-1} \text { a.i. }\right)\end{array}$ & $7 \mathrm{DAE}$ & $14 \mathrm{DAE}$ & $\mathrm{F}_{0}$ & $\mathrm{~F}_{\mathrm{V}} / \mathrm{F}_{\mathrm{M}}$ & ETR & NPQ & \\
\hline 45 & 0 & $0.00 \pm 0.00 \mathrm{AB}$ & $0.00 \pm 0.00 \mathrm{AC}$ & $187.00 \pm 3.89 \mathrm{AB}$ & $0.655 \pm 0.032 \mathrm{AA}$ & $61.91 \pm 11.13 \mathrm{AA}$ & $0.447 \pm 0.057 \mathrm{AB}$ & $0.530 \pm 0.022 \mathrm{AA}$ \\
\hline 30 & 0 & $0.00 \pm 0.00 \mathrm{AB}$ & $0.00 \pm 0.00 \mathrm{AB}$ & $187.00 \pm 3.89 \mathrm{AB}$ & $0.655 \pm 0.032 \mathrm{AA}$ & $61.91 \pm 11.13 \mathrm{AA}$ & $0.447 \pm 0.057 \mathrm{AB}$ & $0.530 \pm 0.022 \mathrm{AA}$ \\
\hline 15 & 0 & $0.00 \pm 0.00 \mathrm{AC}$ & $0.00 \pm 0.00 \mathrm{AB}$ & $187.00 \pm 3.89 \mathrm{AB}$ & $0.655 \pm 0.032 \mathrm{AAB}$ & $61.91 \pm 11.13 \mathrm{AA}$ & $0.447 \pm 0.057 \mathrm{AB}$ & $0.530 \pm 0.022 \mathrm{AA}$ \\
\hline 0 & 0 & $0.00 \pm 0.00 \mathrm{AB}$ & $0.00 \pm 0.00 \mathrm{AB}$ & $187.00 \pm 3.89 \mathrm{AA}$ & $0.655 \pm 0.032 \mathrm{AAB}$ & $61.91 \pm 11.13 \mathrm{AA}$ & $0.447 \pm 0.057 \mathrm{AB}$ & $0.530 \pm 0.022 \mathrm{AA}$ \\
\hline 45 & 3.6 & $2.25 \pm 0.25 \mathrm{AA}$ & $2.33 \pm 0.24 \mathrm{AAB}$ & $232.50 \pm 32.34 \mathrm{AA}$ & $0.662 \pm 0.027 \mathrm{AA}$ & $63.92 \pm 6.60 \mathrm{AA}$ & $1.166 \pm 0.081 \mathrm{AA}$ & $0.433 \pm 0.016 \mathrm{AAB}$ \\
\hline 30 & 3.6 & $3.00 \pm 1.22 \mathrm{AA}$ & $2.00 \pm 0.71 \mathrm{AAB}$ & $206.50 \pm 8.17 \mathrm{AAB}$ & $0.665 \pm 0.037 \mathrm{AA}$ & $66.10 \pm 6.73 \mathrm{AA}$ & $0.478 \pm 0.084 \mathrm{BB}$ & $0.518 \pm 0.029 \mathrm{AA}$ \\
\hline 15 & 3.6 & $2.00 \pm 0.71 \mathrm{AB}$ & $3.75 \pm 1.75 \mathrm{AA}$ & $209.25 \pm 9.67 \mathrm{AB}$ & $0.649 \pm 0.040 \mathrm{AAB}$ & $69.43 \pm 7.20 \mathrm{AA}$ & $0.499 \pm 0.034 \mathrm{BB}$ & $0.498 \pm 0.031 \mathrm{AAB}$ \\
\hline 0 & 3.6 & $2.75 \pm 0.48 \mathrm{AA}$ & $2.75 \pm 0.75 \mathrm{AA}$ & $188.25 \pm 5.23 \mathrm{AA}$ & $0.688 \pm 0.021 \mathrm{AAB}$ & $65.66 \pm 4.67 \mathrm{AA}$ & $0.659 \pm 0.024 \mathrm{BB}$ & $0.526 \pm 0.032 \mathrm{AA}$ \\
\hline 45 & 5.4 & $1.75 \pm 0.63 \mathrm{BAB}$ & $1.33 \pm 0.47 \mathrm{BBC}$ & $238.25 \pm 32.82 \mathrm{AA}$ & $0.665 \pm 0.031 \mathrm{ABA}$ & $60.72 \pm 5.63 \mathrm{AA}$ & $1.122 \pm 0.114 \mathrm{AA}$ & $0.498 \pm 0.040 \mathrm{AAB}$ \\
\hline 30 & 5.4 & $3.00 \pm 0.71 \mathrm{ABA}$ & $3.25 \pm 0.63 \mathrm{ABA}$ & $203.00 \pm 12.36 \mathrm{AAB}$ & $0.702 \pm 0.031 \mathrm{AA}$ & $63.95 \pm 4.91 \mathrm{AA}$ & $0.753 \pm 0.025 \mathrm{AAB}$ & $0.462 \pm 0.030 \mathrm{AAB}$ \\
\hline 15 & 5.4 & $4.75 \pm 1.11 \mathrm{AA}$ & $4.25 \pm 1.44 \mathrm{AA}$ & $195.00 \pm 2.48 \mathrm{AB}$ & $0.631 \pm 0.011 \mathrm{BB}$ & $53.55 \pm 7.09 \mathrm{AA}$ & $0.746 \pm 0.075 \mathrm{AB}$ & $0.364 \pm 0.051 \mathrm{BC}$ \\
\hline 0 & 5.4 & $2.00 \pm 0.00 \mathrm{BA}$ & $2.00 \pm 0.71 \mathrm{ABAB}$ & $209.67 \pm 11.55 \mathrm{AA}$ & $0.680 \pm 0.021 \mathrm{ABAB}$ & $58.40 \pm 4.50 \mathrm{AA}$ & $0.884 \pm 0.223 \mathrm{AB}$ & $0.512 \pm 0.038 \mathrm{AA}$ \\
\hline 45 & 7.2 & $3.50 \pm 0.50 \mathrm{AA}$ & $2.75 \pm 0.75 \mathrm{AAB}$ & $224.25 \pm 24.16 \mathrm{ABAB}$ & $0.635 \pm 0.055 \mathrm{AA}$ & $61.30 \pm 9.20 \mathrm{AA}$ & $1.018 \pm 0.142 \mathrm{AA}$ & $0.475 \pm 0.027 \mathrm{AAB}$ \\
\hline 30 & 7.2 & $4.00 \pm 0.58 \mathrm{AA}$ & $2.50 \pm 0.29 \mathrm{AA}$ & $244.75 \pm 18.32 \mathrm{AA}$ & $0.677 \pm 0.022 \mathrm{AA}$ & $67.13 \pm 8.75 \mathrm{AA}$ & $1.085 \pm 0.070 \mathrm{AA}$ & $0.372 \pm 0.013 \mathrm{BB}$ \\
\hline 15 & 7.2 & $3.00 \pm 0.71 \mathrm{AAB}$ & $3.75 \pm 1.44 \mathrm{AA}$ & $258.50 \pm 8.03 \mathrm{AA}$ & $0.695 \pm 0.019 \mathrm{AA}$ & $72.31 \pm 6.03 \mathrm{AA}$ & $1.198 \pm 0.197 \mathrm{AA}$ & $0.423 \pm 0.034 \mathrm{ABBC}$ \\
\hline 0 & 7.2 & $2.75 \pm 1.60 \mathrm{AA}$ & $2.00 \pm 0.71 \mathrm{AAB}$ & $190.00 \pm 6.72 \mathrm{BA}$ & $0.653 \pm 0.017 \mathrm{AB}$ & $51.94 \pm 6.28 \mathrm{AA}$ & $0.561 \pm 0.048 \mathrm{BB}$ & $0.459 \pm 0.029 \mathrm{ABA}$ \\
\hline 45 & 9.0 & $2.50 \pm 0.29 \mathrm{AA}$ & $4.00 \pm 0.58 \mathrm{AA}$ & $200.75 \pm 13.17 \mathrm{ABAB}$ & $0.668 \pm 0.038 \mathrm{AA}$ & $53.53 \pm 3.88 \mathrm{AA}$ & $0.944 \pm 0.328 \mathrm{BA}$ & $0.399 \pm 0.039 \mathrm{AB}$ \\
\hline 30 & 9.0 & $3.00 \pm 0.71 \mathrm{AA}$ & $3.50 \pm 0.50 \mathrm{AA}$ & $227.50 \pm 21.66 \mathrm{AAB}$ & $0.662 \pm 0.025 \mathrm{AA}$ & $65.32 \pm 3.62 \mathrm{AA}$ & $0.897 \pm 0.310 \mathrm{BAB}$ & $0.418 \pm 0.028 \mathrm{AB}$ \\
\hline 15 & 9.0 & $4.25 \pm 0.48 \mathrm{AA}$ & $3.50 \pm 0.65 \mathrm{AA}$ & $180.00 \pm 0.41 \mathrm{BB}$ & $0.708 \pm 0.015 \mathrm{AA}$ & $61.61 \pm 8.76 \mathrm{AA}$ & $0.768 \pm 0.185 \mathrm{BB}$ & $0.413 \pm 0.012 \mathrm{ABC}$ \\
\hline 0 & 9.0 & $2.33 \pm 0.24 \mathrm{AA}$ & $2.25 \pm 0.25 \mathrm{AAB}$ & $217.33 \pm 12.23 \mathrm{ABA}$ & $0.716 \pm 0.034 \mathrm{AA}$ & $64.94 \pm 4.47 \mathrm{AA}$ & $1.632 \pm 0.220 \mathrm{AA}$ & $0.497 \pm 0.065 \mathrm{AA}$ \\
\hline 45 & & $2.00 \pm 0.31 \mathrm{~A}$ & $2.08 \pm 0.36 \mathrm{AB}$ & $216.55 \pm 10.56 \mathrm{~A}$ & $0.657 \pm 0.015 \mathrm{~A}$ & $60.27 \pm 3.18 \mathrm{~A}$ & $0.939 \pm 0.091 \mathrm{~A}$ & $0.467 \pm 0.016 \mathrm{AB}$ \\
\hline 30 & & $2.60 \pm 0.43 \mathrm{~A}$ & $2.25 \pm 0.35 \mathrm{AB}$ & $213.75 \pm 7.36 \mathrm{~A}$ & $0.672 \pm 0.012 \mathrm{~A}$ & $64.88 \pm 3.02 \mathrm{~A}$ & $0.732 \pm 0.082 \mathrm{~B}$ & $0.460 \pm 0.017 \mathrm{~B}$ \\
\hline 15 & & $2.80 \pm 0.48 \mathrm{~A}$ & $3.05 \pm 0.60 \mathrm{~A}$ & $205.95 \pm 6.85 \mathrm{~A}$ & $0.668 \pm 0.012 \mathrm{~A}$ & $63.76 \pm 3.61 \mathrm{~A}$ & $0.732 \pm 0.080 \mathrm{~B}$ & $0.446 \pm 0.019 \mathrm{~B}$ \\
\hline 0 & & $1.97 \pm 0.38 \mathrm{~A}$ & $1.80 \pm 0.31 \mathrm{~B}$ & $198.45 \pm 4.36 \mathrm{~A}$ & $0.678 \pm 0.011 \mathrm{~A}$ & $60.60 \pm 2.83 \mathrm{~A}$ & $0.837 \pm 0.110 \mathrm{AB}$ & $0.505 \pm 0.017 \mathrm{~A}$ \\
\hline 0 & & $0.00 \pm 0.00 \mathrm{~B}$ & $0.00 \pm 0.00 \mathrm{~B}$ & $187.00 \pm 1.74 \mathrm{C}$ & $0.655 \pm 0.014 \mathrm{~B}$ & $61.91 \pm 4.98 \mathrm{~A}$ & $0.447 \pm 0.026 \mathrm{C}$ & $0.530 \pm 0.010 \mathrm{~A}$ \\
\hline 3.6 & & $2.50 \pm 0.35 \mathrm{~A}$ & $2.71 \pm 0.49 \mathrm{~A}$ & $209.13 \pm 8.84 \mathrm{AB}$ & $0.666 \pm 0.015 \mathrm{AB}$ & $66.28 \pm 2.90 \mathrm{~A}$ & $0.700 \pm 0.077 \mathrm{~B}$ & $0.494 \pm 0.016 \mathrm{AB}$ \\
\hline 5.4 & & $2.88 \pm 0.45 \mathrm{~A}$ & $2.71 \pm 0.49 \mathrm{~A}$ & $211.48 \pm 9.28 \mathrm{AB}$ & $0.669 \pm 0.013 \mathrm{AB}$ & $59.16 \pm 2.70 \mathrm{~A}$ & $0.876 \pm 0.071 \mathrm{AB}$ & $0.459 \pm 0.023 \mathrm{BC}$ \\
\hline 7.2 & & $3.31 \pm 0.44 \mathrm{~A}$ & $2.75 \pm 0.43 \mathrm{~A}$ & $229.38 \pm 9.79 \mathrm{~A}$ & $0.665 \pm 0.016 \mathrm{AB}$ & $63.17 \pm 3.96 \mathrm{~A}$ & $0.965 \pm 0.085 \mathrm{~A}$ & $0.432 \pm 0.016 \mathrm{C}$ \\
\hline 9.0 & & $3.02 \pm 0.28 \mathrm{~A}$ & $3.31 \pm 0.28 \mathrm{~A}$ & $206.40 \pm 7.82 \mathrm{BC}$ & $0.689 \pm 0.014 \mathrm{~A}$ & $61.35 \pm 2.78 \mathrm{~A}$ & $1.060 \pm 0.148 \mathrm{~A}$ & $0.432 \pm 0.021 \mathrm{C}$ \\
\hline \multicolumn{9}{|c|}{ Variation source } \\
\hline Applicatic & time $(\mathrm{T})$ & 0.1363 & 0.0672 & 0.2062 & 0.3856 & 0.6909 & 0.093 & 0.0369 \\
\hline Rate (R) & & $<0.001$ & $<0.001$ & 0.0041 & 0.2135 & 0.7297 & $<0.001$ & $<0.001$ \\
\hline $\mathrm{T} \times \mathrm{R}$ & & 0.3031 & 0.6058 & 0.0444 & 0.1961 & 0.9171 & $<0.001$ & 0.0929 \\
\hline Block & & 0.8446 & 0.2577 & 0.1012 & $<0.001$ & 0.0972 & 0.2395 & 0.3357 \\
\hline CV (\%) & & 58.14 & 65.81 & 13.95 & 5.96 & 23.58 & 36.66 & 13.80 \\
\hline
\end{tabular}

${ }^{(1)}$ Means followed by equal letters, lowercase letters compare application times and uppercase letter compare the different rates applied, do not differ by Duncan's test, at $5 \%$ probability. ${ }^{(2)}$ Days before soybean sowing. ${ }^{(3)} \mathrm{SDM}$, shoot dry matter. CV, coefficient of variation.

\section{Conclusions}

1. The use of metsulfuron-methyl has negative effects on the NA 5909 RG soybean (Glycine max) cultivar, mainly when applied closer to soybean sowing ( 0 day before sowing) and at highest rate (9.0 $\mathrm{g} \mathrm{ha}^{-1}$ a.i.).
2. Metsulfuron-methyl drastically reduces the photosynthetic electron transport rate when applied at rates greater than $3.6 \mathrm{~g} \mathrm{ha}^{-1}$ a.i. and in periods less than 45 days from soybean sowing, whereas nonphotochemical quenching increases in soybean plants exposed to a herbicide carryover of up to $5.4 \mathrm{~g} \mathrm{ha}^{-1}$ a.i. 
3. Metsulfuron-methyl carryover reduces soybean 1,000-grain weight and productivity as a function of the application rate (higher than $5.4 \mathrm{~g} \mathrm{~h}^{-1}$ a.i.) and sowing period (intervals shorter than 15 days after herbicide application).

4. Cucumber (Cucumis sativus), used as a bioindicator plant, confirms the negative effects of metsulfuron-methyl on physiological traits, regardless of the rate applied until 45 days before sowing.

5. The use of metsulfuron-methyl is not indicated safely in a period of less than 45 days before soybean sowing, regardless of the applied rate.

\section{Acknowledgments}

To Universidade Federal da Fronteira Sul (UFFS) and to Conselho Nacional de Desenvolvimento Científico e Tecnológico (CNPq), for financial support (process numbers PES-2018-0884 and 406221/20162, respectively); and to Fundação de Amparo à Pesquisa do Estado do Rio Grande do Sul (Fapergs), to Coordenação de Aperfeiçoamento de Pessoal de Nível Superior (Capes), and to Conselho Nacional de Desenvolvimento Científico e Tecnológico (CNPq), for fellowships (process numbers Fapergs/Capes 05/2017, Capes/PNPD 88887.352933/2019-00, and CNPq/PQ1D 306927/2019-5).

\section{References}

AGOSTINETTO, D.; FRAGA, D.S.; VARGAS, L.; OLIVEIRA, A.C.B.; ANDRES, A.; VILLELA, F.A. Response of soybean cultivars in rotation with irrigated rice crops cultivated in Clearfield ${ }^{\circledR}$ system. Planta Daninha, v.36, e018170991, 2018. DOI: https://doi.org/10.1590/S0100-83582018360100048.

AGOSTINETTO, D.; PERBONI, L.T.; LANGARO, A.C.; GOMES, J.; FRAGA, D.S.; FRANCO, J.J. Changes in photosynthesis and oxidative stress in wheat plants submitted to herbicides application. Planta Daninha, v.34, p.1-9, 2016. DOI: https://doi.org/10.1590/S0100-83582016340100001.

AISENBERG, G.R.; FRAGA, D.S.; SILVA, B.M. da; ULGUIM, A. da R.; SILVA, J.D.G. da; AGOSTINETTO, D. Weed control and selectivity of alternative herbicides to glyphosate in soy cultivation. International Journal of Current Research, v.8, p.37729-37735, 2016

BAKER, N.R.; ROSENQVIST, E. Applications of chlorophyll fluorescence can improve crop production strategies: an examination of future possibilities. Journal of Experimental Botany, v.55, p.1607-1621, 2004. DOI: https://doi.org/10.1093/jxb/erh196.

BALBINOT JUNIOR, A.A.; OLIVEIRA, M.C.N. de; FRANCHINI, J.C.; DEBIASI, H.; ZUCARELI, C.; FERREIRA, A.S.; WERNER, F. Phenotypic plasticity in a soybean cultivar with indeterminate growth type. Pesquisa Agropecuária Brasileira, v.53, p.1038-1044, 2018. DOI: https://doi.org/10.1590/ S0100-204X2018000900007.

BENBROOK, C.M. Trends in glyphosate herbicide use in the United States and globally. Environmental Sciences Europe, v.28, art.3, 2016. DOI: https://doi.org/10.1186/s12302-016-0070-0.

COMIN, R.C.; MENEGHELLO, G.E.; FRANCO, J.J.; LEVIEN, A.M.; KEHL, K.; COMIN, G.C. Qualidade fisiológica de sementes de soja submetidas à dessecação em pré-colheita. Colloquium Agrariae, v.14, p.112-120, 2018. DOI: https://doi.org/10.5747/ca.2018.v14.n4.a254.

CRUZ, S.C.S.; SENA JUNIOR, D.G. de; SANTOS, D.M.A. dos; LUNEZZO, L.O.; MACHADO, C.G. Cultivo de soja sob diferentes densidades de semeadura e arranjos espaciais. Revista de Agricultura Neotropical, v.3, p.1-6, 2016. DOI: https://doi.org/10.32404/rean.v3i1.431.

DAS, A.; ELDAKAK, M.; PAUDEL, B.; KIM, D.-W.; HEMMATI, H.; BASU, C.; ROHILA, J.S. Leaf proteome analysis reveals prospective drought and heat stress response mechanisms in soybean. BioMed Research International, v.2016, art.6021047, 2016. DOI: https://doi.org/10.1155/2016/6021047.

DUKE, S.O. Why have no new herbicide modes of action appeared in recent years? Pest Management Science, v.68, p.505-512, 2012. DOI: https://doi.org/10.1002/ps.2333.

FERREIRA, A.S.; BALBINOT JUNIOR, A.A.; WERNER, F.; ZUCARELI, C.; FRANCHINI, J.C.; DEBIASI, H. Plant density and mineral nitrogen fertilization influencing yield, yield components and concentration of oil and protein in soybean grains. Bragantia, v.75, p.362-370, 2016. DOI: https://doi.org/10.1590/1678-4499.479.

FILIZOLA, H.F.; FERRACINI, V.L.; SANS, L.M.A.; GOMES, M.A.F.; FERREIRA, C.J.A. Monitoramento e avaliação do risco de contaminação por pesticidas em água superficial e subterrânea na região de Guaíra. Pesquisa Agropecuária Brasileira, v.37, p.659-667, 2002. DOI: https://doi.org/10.1590/s0100$204 \times 2002000500011$.

FRAGA, D.S.; AGOSTINETTO, D.; LANGARO, A.C.; OLIVEIRA, C.; ULGUIM, A.R.; SILVA, J.D.G. Morphological and metabolic changes in soybean plants cultivated in irrigated rice rotation and as affected by imazapyr and imazapic herbicides carryover. Planta Daninha, v.37, e019165375, 2019. DOI: https://doi.org/10.1590/s0100-83582019370100023.

GALON, L.; FERREIRA, F.A.; SILVA, A.A.; CONCENÇO, G.; FERREIRA, E.A.; BARBOSA, M.H.P.; SILVA, A.F.; ASPIAZÚ, I.; FRANÇA, A.C.; TIRONI, S.P. Influência de herbicidas na atividade fotossintética de genótipos de cana-de-açúcar. Planta Daninha, v.28, p.591-597, 2010. DOI: https://doi.org/10.1590/ s0100-83582010000300016.

GENTY, B.; BRIANTAIS, J.-M.; BAKER, N.R. The relationship between the quantum yield of photosynthetic electron transport and quenching of chlorophyll fluorescence. Biochimica et Biophysica Acta (BBA) - General Subjects, v.990, p.87-92, 1989. DOI: https://doi.org/10.1016/S0304-4165(89)80016-9.

GILBERT, N. A hard look at GM crops. Nature, v.497, p.24-26, 2013. DOI: https://doi.org/10.1038/497024a. 
GONÇALVES, F.A.R.; MELO, C.A.D.; QUEIROZ, P.C. de; ENDO, R.T.; SILVA, D.V. da; REIS, M.R. dos. Atividade residual de herbicidas nas culturas do milho e da soja. Revista de Ciências Agrárias, v.61, p.1-6, 2018. DOI: https://doi.org/10.22491/ rca.2018.2570.

ISMAIL, B.S.; AZLIZAN, B.A. Persistence and bioactivity of metsulfuron-methyl in three soils. Journal of Environmental Science and Health, Part B, v.37, p.345-353, 2002. DOI: https://doi.org/10.1081/PFC-120004475.

LAMEGO, F.P.; KASPARY, T.E.; RUCHEL, Q.; GALLON, M.; BASSO, C.J.; SANTI, A.L. Manejo de Conyza bonariensis resistente ao glyphosate: coberturas de inverno e herbicidas em pré-semeadura da soja. Planta Daninha, v.31, p.433-442, 2013. DOI: https://doi.org/10.1590/S0100-83582013000200022.

MANUAL de calagem e adubação para os Estados do Rio Grande do Sul e de Santa Catarina. 11.ed. [Porto Alegre]: Comissão de Química e Fertilidade do Solo - RS/SC, 2016. 375p.

MARINHO, M.I. da C.; COSTA, A.I.G.; VIEIRA, N.M.; PAIVA, M.C.G.; FREITAS, F.C.L. de; SILVA, A.A. da. Validation and application of a QuEChERS based method for estimation of half-life of imidazolinone herbicides in soils by LC-ESI-MS/ MS. Ecotoxicology and Environmental Safety, v.167, p.212-217, 2019. DOI: https://doi.org/10.1016/j.ecoenv.2018.09.075.

MATOS, A.K.A. de; CARBONARI, C.A.; GOMES, G.L.G.C.; VELINI, E.D. Dynamics of preemergent herbicides in production systems with straw. Revista Brasileira de Herbicidas, v.15, p.97106, 2016. DOI: https://doi.org/10.7824/rbh.v15i1.441.

MELO, C.A.D.; DIAS, R. de C.; MENDES, K.F.; ASSIS, A.C. de L.P.; REIS, M.R. dos. Herbicides carryover in systems cultivated with vegetable crops. Revista Brasileira de Herbicidas, v.15, p.67-78, 2016. DOI: https://doi.org/10.7824/rbh.v15i1.434.

NIYOGI, K.K.; BJÖRKMAN, O.; GROSSMAN, A.R. The roles of specific xanthophylls in photoprotection. Proceedings of the National Academy of Sciences of the United States of America, v.94, p.14162-14167, 1997. DOI: https://doi.org/10.1073/ pnas.94.25.14162.

NORSWORTHY, J.K.; WARD, S.M.; SHAW, D.R.; LLEWELLYN, R.S.; NICHOLS, R.L.; WEBSTER, T.M.; BRADLEY, K.W.; FRISVOLD, G.; POWLES, S.B.; BURGOS, N.R.; WITT, W.W.; BARRETT, M. Reducing the risks of herbicide resistance: best management practices and recommendations. Weed Science, v.60, p.31-62, 2012. DOI: https://doi.org/10.1614/ ws-d-11-00155.1.

NTOANIDOU, S.; KALOUMENOS, N.; DIAMANTIDIS, G.; MADESIS, P.; ELEFTHEROHORINOS, I. Molecular basis of Cyperus difformis cross-resistance to ALS-inhibiting herbicides. Pesticide Biochemistry and Physiology, v.127, p.38-45, 2016. DOI: https://doi.org/10.1016/j.pestbp.2015.09.004.

OLIVEIRA, T.L. de; SENOSKI, M. de P.; ASSIS, A.C. de L.P.; MIRANDA, V.P. de; MELO, C.A.D.; REIS, M.R. dos. Seleção de espécies bioindicadoras do herbicida ethoxysulfuron. Revista de Ciências Agrárias, v.61, p.1-8, 2018. DOI: https://doi.org/10.22491/rca.2018.2613.

PEEL, M.C.; FINLAYSON, B.L.; MCMAHON, T.A. Updated world map of the Köppen-Geiger climate classification.
Hydrology and Earth System Sciences, v.11, p.1633-1644, 2007. DOI: https://doi.org/10.5194/hess-11-1633-2007.

PEROTTI, V.E.; LARRAN, A.S.; PALMIERI, V.E.; MARTINATTO, A.K.; PERMINGEAT, H.R. Herbicide resistant weeds: a call to integrate conventional agricultural practices, molecular biology knowledge and new technologies. Plant Science, v.290, 110255, 2020. DOI: https://doi.org/10.1016/j. plantsci.2019.110255.

PLACIDO, H.F.; ALBRECHT, L.P.; KRENCHINSKI, F.H.; ALBRECHT, A.J.P.; KORBER, A.H.C.; TESSELE, A.; LORENZETTI, J.B. Desiccants application season in preemergence in conventional soybeans. Revista Brasileira de Herbicidas, v.14, p.93-102, 2015. DOI: 10.7824/rbh.v14i2.297.

R CORE TEAM. R: a language and environment for statistical computing. Vienna: R Foundation for Statistical Computing, 2013.

RODRIGUES, B.N.; ALMEIDA, F.S. de. Guia de herbicidas. 7.ed. Londrina: Produção Independente, 2018. 764p.

SANTOS, C.G.; REIS, A.N.; FERREIRA, A.C.; MARQUES, R.P. Residual do herbicida imazetapir na cultura do milho. Colloquium Agrariae, v.14, p.154-159, 2018a. DOI: https://doi.org/10.5747/ca.2018.v14.n3.a237.

SANTOS, H.G. dos; JACOMINE, P.K.T.; ANJOS, L.H.C. dos; OLIVEIRA, V.Á. de; LUMBRERAS, J.F.; COELHO, M.R.; ALMEIDA, J.A. de; ARAÚJO FILHO, J.C. de; OLIVEIRA, J.B. de; CUNHA, T.J.F. Sistema brasileiro de classificação de solos. 5.ed. rev. e ampl. Brasília: Embrapa, 2018b. 356p.

SANTOS, T.T.M. dos; TIMOSSI, P.C.; LIMA, S.F.; GONÇALVES, D.C.; SANTANA, M.V. Associação dos herbicidas diclosulam e glyphosate na dessecação visando o controle residual de plantas daninhas na cultura da soja. Revista Brasileira de Herbicidas, v.15, p.138-147, 2016. DOI: https://doi.org/10.7824/rbh.v15i2.460.

SOLTANI, N.; MASHHADI, H.R.; MESGARAN, M.B.; COWBROUGH, M.; TARDIF, F.J.; CHANDLER, K.; NURSE, R.E.; SWANTON, C.J.; SIKKEMA, P.H. The effect of residual corn herbicides on injury and yield of soybean seeded in the same season. Canadian Journal of Plant Science, v.91, p.571-576, 2011. DOI: https://doi.org/10.4141/cjps10110.

SOUZA, V.Q. de; BELLÉ, R.; FERRARI, M.; PELEGRIN, A.J. de; CARON, B.O.; NARDINO, M.; FOLLMANN, D.N.; CARVALHO, I.R. Componentes de rendimento em combinações de fungicidas e inseticidas e análise de trilha em soja. Global Science and Technology, v.8, p.167-176, 2015. DOI: https://doi.org/10.14688/1984-3801/gst.v8n1p167-176.

SU, W.C.; SUN, L.L.; GE, Y.H.; WU, R.H.; XU, H.L.; LU, C.T. The residual effects of bensulfuron-methyl on growth and photosynthesis of soybean and peanut. Photosynthetica, v.56, p.670-677, 2018. DOI: https://doi.org/10.1007/s11099-017-0726-z.

SUN, L.; XU, H.; HAO, H.; AN, S.; LU, C.; WU, R.; SU, W. Effects of bensulfuron-methyl residue on photosynthesis and chlorophyll fluorescence in leaves of cucumber seedlings. PLoS ONE, v.14, e0215486, 2019. DOI: https://doi.org/10.1371/journal. pone. 0215486 .

TIMM, S.; FLORIAN, A.; FERNIE, A.R.; BAUWE, H. The regulatory interplay between photorespiration and photosynthesis. 
Journal of Experimental Botany, v.67, p.2923-2929, 2016. DOI: https://doi.org/10.1093/jxb/erw083.

VELINI, E.D.; OSIPE, R.; GAZZIERO, D.L.P. (Coord.). Procedimentos para instalação, avaliação e análise de experimentos com herbicidas. Londrina: SBCPD, 1995. 42p.
YANG, J.-H.; CUI, C.-X.; QU, L.-B.; CHEN, J.; ZHOU, X.-M.; ZHANG, Y.-P. Preparation of a monolithic magnetic stir bar for the determination of sulfonylurea herbicides coupled with HPLC. Microchemical Journal, v.141, p.369-376, 2018. DOI: https://doi.org/10.1016/j.microc.2018.05.049. 